\title{
Histone demethylase KDM2B regulates lineage commitment in normal and malignant hematopoiesis
}

\author{
Jaclyn Andricovich, ${ }^{1,2}$ Yan Kai, ${ }^{1,2,3}$ Weiqun Peng, ${ }^{3}$ Adlen Foudi, ${ }^{4}$ and Alexandros Tzatsos ${ }^{1,2}$ \\ ${ }^{1}$ Cancer Epigenetics Laboratory, Department of Anatomy and Regenerative Biology, and ${ }^{2}$ Ceorge Washington University (GWU) Cancer Center, GWU School of Medicine and Health Sciences, \\ Washington, DC, USA. ${ }^{3}$ Department of Physics, GWU, Washington DC, USA. ${ }^{4}$ INSERM UMR-S935, André Lwoff Institute, Paul Brousse Hospital, Villejuif, France.
}

\begin{abstract}
The development of the hematopoietic system is a dynamic process that is controlled by the interplay between transcriptional and epigenetic networks to determine cellular identity. These networks are critical for lineage specification and are frequently dysregulated in leukemias. Here, we identified histone demethylase KDM2B as a critical regulator of definitive hematopoiesis and lineage commitment of murine hematopoietic stem and progenitor cells (HSPCs). RNA sequencing of $K d m 2 b$-null HSPCs and genome-wide ChIP studies in human leukemias revealed that KDM2B cooperates with polycomb and trithorax complexes to regulate differentiation, lineage choice, cytokine signaling, and cell cycle. Furthermore, we demonstrated that KDM2B exhibits a dichotomous role in hematopoietic malignancies. Specifically, we determined that KDM2B maintains lymphoid leukemias, but restrains RAS-driven myeloid transformation. Our study reveals that KDM2B is an important mediator of hematopoietic cell development and has opposing roles in tumor progression that are dependent on cellular context.
\end{abstract}

\section{Introduction}

Hematopoietic stem and progenitor cells (HSPCs) possess the capacity to self renew and to produce all hematopoietic lineages upon differentiation. In mice, these progenitors reside in a compartment immunophenotypically defined by lack of lineage-specific markers (lineage negative, $\mathrm{Lin}^{\mathrm{neg}}$ ) and positive for both c-Kit and Sca-1 (hereafter $\mathrm{Lin}^{\text {neg }} \mathrm{KS}^{+}$). Use of CD48 and CD150 further identified a population of long-term hematopoietic stem cells $\left(\mathrm{Lin}^{\text {neg }} \mathrm{KS}^{+} \mathrm{CD} 48^{-} \mathrm{CD} 150^{+}\right.$, hereafter LT-HSCs) that rescue myeloablated mice from hematopoietic failure and establish long-term multilineage reconstitution (1). During embryogenesis, primitive hematopoiesis initiates in the yolk sac around E7.5 and gives rise to erythromyeloid progenitors; around E10.5, definitive hematopoiesis commences from the hemogenic endothelium of the aorta-gonad-mesonephros (AGM) region, demarcated by VE-cadherin and CD45 surface markers. The hematopoietic cells that arise from the AGM are the first HSPCs to harbor multilineage reconstitution capacity. These HSPCs subsequently migrate and expand in the fetal liver prior to homing the BM by E16.5, the permanent site for hematopoiesis throughout adulthood $(2-4)$, where they are maintained in a quiescent state $(5,6)$.

Hematopoietic development is dynamically controlled by the interplay of transcriptional and epigenetic networks to determine cellular identity. Deregulation of those networks frequently occurs in hematopoietic malignancies, causing accumulation of immature progenitors, and facilitates cell transformation in the context of additional genetic and epigenetic changes (3). Polycomb (PcG) and trithorax (TrxG) group proteins form evolutionary conserved, multiprotein complexes that have been shown to epigenetically coordinate proliferation and differentiation throughout hematopoiesis. In mammalian cells, the TrxG protein MLL1 resides in a complex with

Conflict of interest: The authors have declared that no conflict of interest exists. Submitted: July 31, 2015; Accepted: December 8, 2015.

Reference information: J Clin Invest. 2016;126(3):905-920. doi:10.1172/JCI84014.
WDR5, RBBP5, and ASH2L that methylates histone H3K4 to activate transcription (7). On the other hand, PcG proteins function as global transcriptional repressors that antagonize TrxG through histone H3K27 methylation and H2AK119 ubiquitination. PcG proteins form 2 distinct complexes: polycomb repressive complex 2 (PRC2) contains SUZ12, EED, and EZH2, which methylates H3K27 to recruit polycomb repressive complex 1 (PRC1) consisting of the catalytic subunit RING1B as well as PCGF and CBX proteins (8). Deregulation of TrxG and PcG proteins is a common defect in hematopoietic malignancies. For instance, various chromosomal translocations have been characterized for the $M L L 1$ gene in pediatric leukemias (9). On the other hand, PRC2 components exhibit loss-of-function mutations in myelodysplastic syndrome (MDS), acute myeloid leukemia (AML), and a subset of adult T cell acute lymphoblastic leukemias (T-ALL) (10-15) as well as gain-of-function mutations in follicular and diffuse large B cell lymphoma (16-18). Thus, elucidating the crosstalk between PcG and TrxG proteins will provide insight for both hematopoietic homeostasis and malignancies.

We previously discovered KDM2B (also known as Ndy1 and Fbxl10), a Jumonji (JmjC) domain histone H3K36 di-demethylase (H3K36me2), following a genome-wide insertional mutagenesis screen in Moloney murine leukemia virus-induced (MoMuLVinduced) T cell lymphomas $(19,20)$. KDM2B is upregulated and exhibits prooncogenic activity through repression of the Ink4a/ Arf locus and other tumor-suppressor pathways (19, 21-23). At the molecular level, KDM2B is an integral component of a noncanonical PRC1 complex and crosstalks with PRC2 to regulate cell proliferation, senescence, oncogenesis, and somatic cell reprogramming $(22,24-27)$. Here, we report the generation of genetically engineered mouse models to conditionally ablate or overexpress KDM2B in the hematopoietic system. We found that KDM2B is required for embryonic development and definitive hematopoiesis. In mice, loss of KDM2B severely compromised the pool of HSPCs and lymphoid differentiation. At the molecular 
A

Chr5

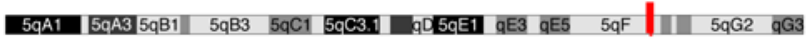

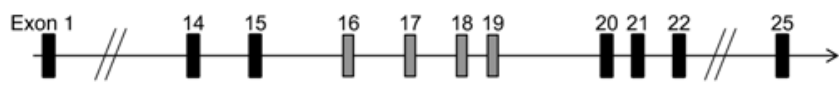

Targeted allele

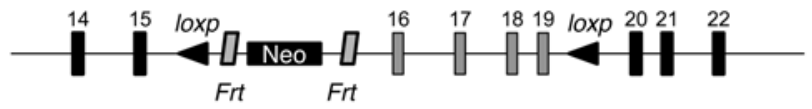

$K d m 2 b^{\text {lox }}$ allele

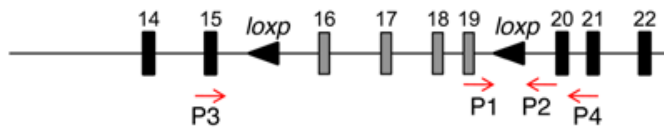

$K d m 2 b^{\star}$ allele

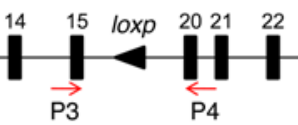

C

Gata1-Cre Kdm2 2b+/+Gata1-Cre Kdm2 $b^{\text {th }}$

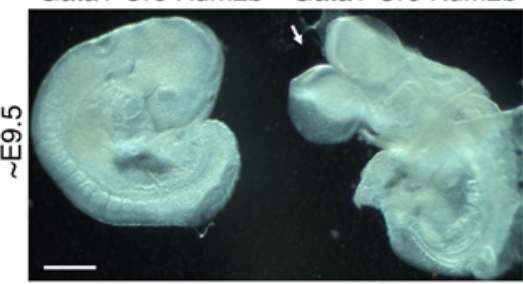

E Tie2-Cre $\mathrm{Kdm} 2 \mathrm{~b}^{+/+}$Tie2-Cre Kdm2 $b^{\prime \prime m}$

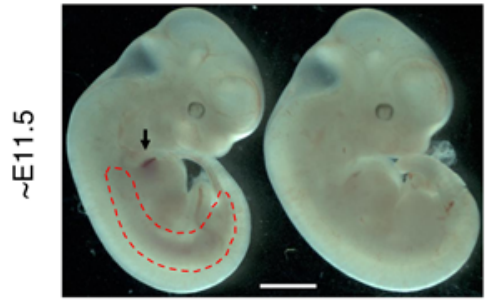

$\mathbf{F}$

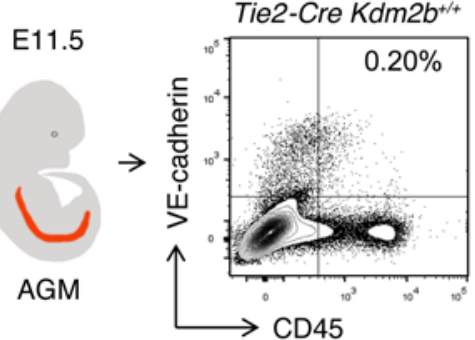

$+F l p$

(1) \begin{tabular}{lll} 
P 4 & 4 \\
\hline
\end{tabular}

Cre

\section{Gata1-Cre Kdm2b+/+}
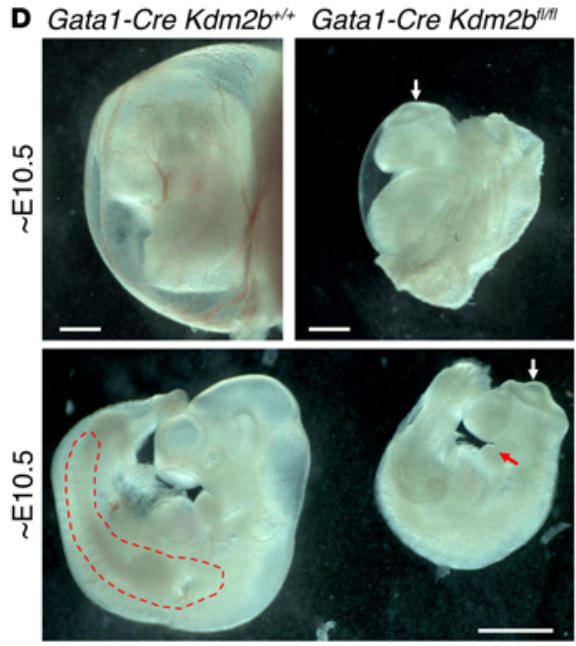

Tie2-Cre Kdm2b

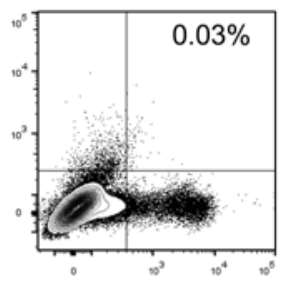

VE-cadherin/CD45

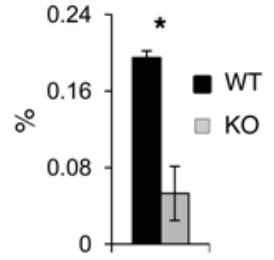

B
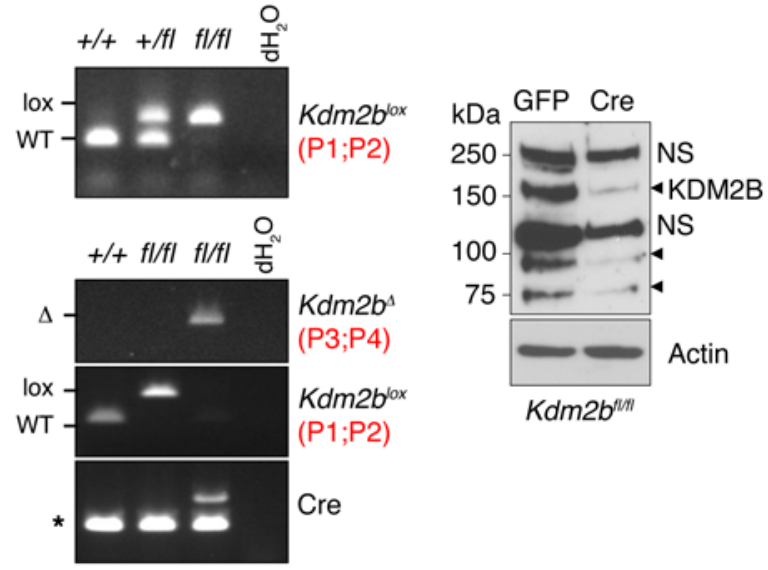

G
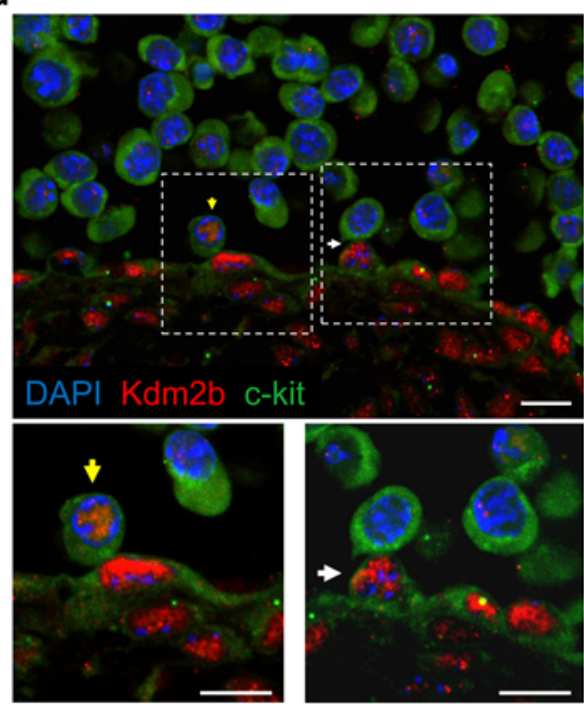

Figure 1. KDM2B is essential for embryonic development. (A) Targeting strategy to generate the $K d m 2 b$ conditional allele. Exons $16-19$ were targeted with loxp sites and a neomycin (Neo) cassette flanked by Frt sites. To remove the Neo cassette and generate the Kdm2 $b^{\text {lox }}$ allele, mice were crossed with the actin-Flpe strain. Tissue-specific deletion was achieved by crossing with appropriate Cre strains. Red arrows depict the position of PCR primers for the detection of $K d m 2 b^{10 x}(\mathrm{P} 1: \mathrm{P} 2)$ and the recombined allele $K d m 2 b^{4}$ (P3:P4). (B) Upper left: representative PCR to detect the $K d m 2 b^{10 x}$ allele in tail-tip DNA samples from WT, heterozygous, and homozygous mice. Bottom left: $K d m 2 b^{f l / f l}$ MEFs infected with adenoviruses that express GFP or Cre recombinase. PCR of genomic DNA shows excision of the floxed allele upon Cre expression. Asterisk indicates internal PCR control. Right: Western blot showing downregulation of long and short isoforms (arrowheads) of KDM2B from MEF whole-cell lysates. NS, nonspecific. (C and D) Gross images of WT and $K d m 2 b$-null embryos at (C) E9.5 and (D) E10.5. Neural tube defects and excencephaly (white arrows), craniofacial defects (red arrow), and lack of yolk sac vasculature were observed in $\mathrm{KO}$ embryos. The red dotted line traces the aorta/AGM. Scale bars: $0.5 \mathrm{~mm}$ (C); $1 \mathrm{~mm}$ (D). (E) Gross images of E11.5 WT and Tie2-Cre $K d m 2 b^{f / f l}$ embryos. Red dotted line traces the aorta/AGM, and the black arrow points to the heart. Scale bar: 2 mm. (F) Flow cytometric analysis of dissected AGM showing the frequency of double-positive VE-cadherin/CD45 cells. Graph shows the mean \pm SEM. $n=3$. ${ }^{*} P<0.05$. (G) Immunofluorescent staining of aortas from E10.5 embryos for KDM2B (red; nuclear) and c-Kit (green; cell surface). White arrow shows budding hemogenic endothelium and yellow arrow a circulating "stem-like" cell. Nuclei were stained with DAPI (blue). Scale bars: $10 \mu \mathrm{m}$. 
level, KDM2B binds both repressed and active chromatin through respective PcG and TrxG complexes to regulate developmental pathways, including NOTCH and WNT, lineage commitment, and proliferation of HSPCs. Finally, we show that KDM2B exhibits opposing roles in hematological malignancies, as it can promote or antagonize tumor progression depending on the cellular context.

\section{Results}

Genetic ablation of $K d m 2 b$ causes embryonic lethality and disrupts development of hematopoiesis. We generated $K d m 2 b^{f / f l}$ conditional KO mice by flanking exons 16-19 with loxp sites (Figure 1, A and $\mathrm{B}$, and Supplemental Figure 1A; supplemental material available online with this article; doi:10.1172/JCI84014DS1). Cre-mediated recombination fuses exon 15 to 20 and deletes all protein coding isoforms of $K d m 2 b$ by generating an out-of-frame transcript (Supplemental Figure 1A). To achieve early embryonic deletion, including germline, $K d m 2 b^{f / f l}$ mice were bred with transgenic mice expressing Cre under the control of the Gata1 promoter (28). Heterozygous mice were viable, fertile, and free of developmental defects. Litters born from heterozygous crosses were smaller in number and deviated from Mendelian ratios, as evident by the absence of Gata1-Cre Kdm2 $b^{f / f l}$ animals (0/54 pups), suggesting that early deletion of $K d m 2 b$ is embryonic lethal. Analysis of Gata1Cre $K d m 2 b^{f l / f l}$ embryos revealed severe developmental abnormalities including smaller size and failure of neural tube closure as well as limb and craniofacial malformation; these embryos died in utero around E11.5-E13.5 (Figure 1, C and D, and Supplemental Figure $1, \mathrm{~B}$ and $\mathrm{C})$, and complete penetrance was observed. These developmental defects coincided with the broad expression of $K d m 2 b$, particularly in neuronal tissues (Supplemental Figure 1D).

We observed that KO embryos and their yolk sacs were pale and lacked vasculature (Figure 1D and Supplemental Figure 1B) suggesting the need for KDM2B in early hematopoietic development. To directly address this question and to exclude adverse effects stemming from germline deletion, we generated crosses with Tie2-Cre transgenic mice to excise $K d m 2 b$ in the hemogenic endothelium (29). Heterozygous $K d m 2 b$ mice were viable, fertile, and born at the expected frequency without signs of compromised hematopoiesis; however, the frequency of delivered Tie2-Cre $K d m 2 b^{f / f l}$ pups deviated from the Mendelian ratio. We recovered 3 viable pups (3/40, $7.5 \%$; expected $25 \%)$; one was notably smaller, exhibited growth retardation, and died within 2 weeks, prohibiting further study. The other 2 grew normally, but we failed to detect recombination of the $K d m 2 b$ locus in genomic DNA isolated from the blood. The incomplete penetrance may be attributed to insufficient recombination when the Tie2-Cre allele is inherited from the male germline (29). At E11.5, Tie2-Cre Kdm2 $b^{f / f l}$ embryos appeared pale and lacked discernible aorta and heart, but did not show other gross abnormalities (Figure 1E). Flow cytometric analysis of the AGM revealed that loss of KDM2B caused a reduction in VEcadherin $^{+} \mathrm{CD} 45^{+}$hemogenic endothelial cells (Figure 1F). Furthermore, immunofluorescent staining of E10.5 WT embryos showed that budding endothelial cells of the dorsal aorta stained positive for both KDM2B and c-Kit (Figure 1G and Supplemental Figure 1E). Of note, circulating cells with "stem-like" morphology (dense chromatin and scant cytoplasm) were also positive for KDM2B (Figure $1 \mathrm{G}$ and Supplemental Figure 1E). Interestingly, metaanalysis of a previously published RNAi screen (30) revealed that knockdown (KD) of $j h d m 1 b$ ( $K d m 2 b$ homolog) in zebrafish compromised both primitive and definitive hematopoiesis (Supplemental Figure $1 \mathrm{~F}$ ) and caused embryonic lethality (Supplemental Figure 1G), suggesting an evolutionary conserved role. Collectively, these data show that KDM2B is required for definitive hematopoiesis.

$K D M 2 B$ is required for maintenance and lineage commitment of hematopoietic stem/progenitors. To further investigate the role of $K d m 2 b$ in hematopoiesis, we used Vav1-Cre transgenic mice to specifically target definitive hematopoietic cells (31). Vav1-Cre Kdm2 $b^{f / f l}$ animals were born at expected Mendelian ratios and were viable, fertile, and free of developmental abnormalities. However, immunophenotypic analysis revealed a 5-fold decrease in the frequency of $\mathrm{Lin}^{\text {neg }} \mathrm{KS}^{+}$and LT-HSCs (Figure 2A). To exclude the possibility that this effect is contingent on deletion early in hematopoiesis, we generated $\mathrm{M} x \mathrm{1}$-Cre $\mathrm{Kdm} 2 \mathrm{~b}^{f / f l}$ mice to allow excision in adult animals upon polyinosinicpolycytidylic acid $(p I p C$ ) administration (Figure 2A and Supplemental Figure 2A). Flow cytometric analysis of $\mathrm{M} x \mathrm{1}$-Cre $K d m 2 b^{f l / f l} \mathrm{BM}$ showed a similar decrease of the $\mathrm{Lin}^{\text {neg }} \mathrm{KS}^{+}$and LT-HSC populations (Figure 2A). These data are consistent with the expression pattern, as KDM2B is highly expressed in murine and human HSPCs compared with other tissues (Supplemental Figure 2, B and C). Moreover, quantitative reverse-transcription PCR (qRT-PCR) revealed that LT-HSCs preferentially express $K d m 2 b$ compared with $K d m 2 a$ (the other known H3K36 di-demethylase) and its levels decrease with differentiation (Supplemental Figure 2D).

LT-HSCs exhibit high self-renewal capacity and produce multipotent progenitors, which differentiate toward either the lymphoid or myeloid lineage (Supplemental Figure 2E). Common lymphoid progenitors (CLPs) are defined as $\mathrm{Lin}^{\text {neg }} \mathrm{K}^{\mathrm{lo}} \mathrm{S}^{\mathrm{lo}} \mathrm{IL}-7 \mathrm{R} \alpha^{+}$ (also known as CD127), whereas the $\mathrm{Lin}^{\mathrm{neg}} \mathrm{K}^{+} \mathrm{S}^{-}$population contains common myeloid progenitors (CMP) and also more committed granulocyte-macrophage progenitors (GMP) and megakaryocyte-erythroid progenitors (MEP), distinguished based on their relative expression of Fc $\gamma$ RIII/II (CD16/32) and CD34. A more primitive population known as lymphoid-primed multipotent progenitors (LMPPs), defined as $\mathrm{Lin}^{\text {neg }} \mathrm{KS}^{+} \mathrm{Flt} 3^{+}$(also known as CD135, Supplemental Figure 2E), has also been described; although harboring multilineage reconstitution potential, LMPPs are biased to differentiate toward the lymphoid lineage (32). Analysis of BM revealed that loss of $K d m 2 b$ dramatically decreased the numbers of both LMPPs and CLPs, suggesting compromised lymphoid specification (Figure 2B). Conversely, loss of $K d m 2 b$ was accompanied by a mild yet significant increase of GMPs, without altering CMPs and MEPs (Figure 2B). BM cytospin preparations confirmed the expansion of myeloid and paucity of lymphoid cells (Figure 2C). This was corroborated by hematopoietic progenitor colonyforming assays, which showed disrupted pre-B cell colony formation together with an increased number of myeloid colonies without altering their relative distribution (Supplemental Figure 2F). Analysis of peripheral blood from Vav1-Cre Kdm2 $b^{f / f l}$ mice showed leukopenia and thrombocytopenia (Figure 2D), which confirmed the role of KDM2B in BM homeostasis. No change in the number of red blood cells ( $\mathrm{rbc}$ ) was observed.

To study the impact of KDM2B overexpression, we generated a triple-transgenic mouse model that combines cre-lox and tet-regulated gene-switch technologies. When crossed with the Vav1-Cre strain, 
A
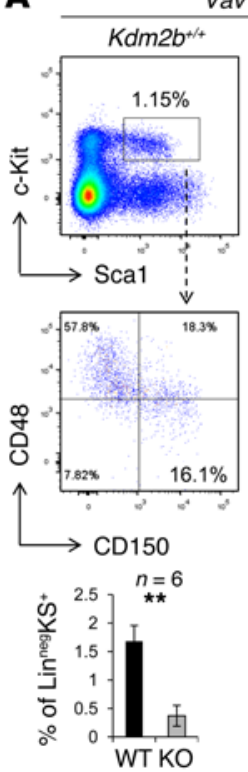

B Vav1-Cre Kdm2 $2 b^{+/ 4}$

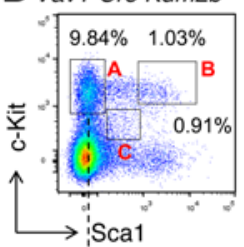

Sca1

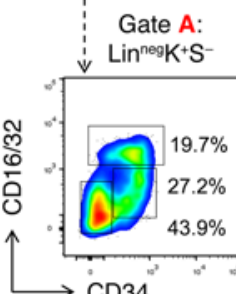

CD34
Kdm2b年
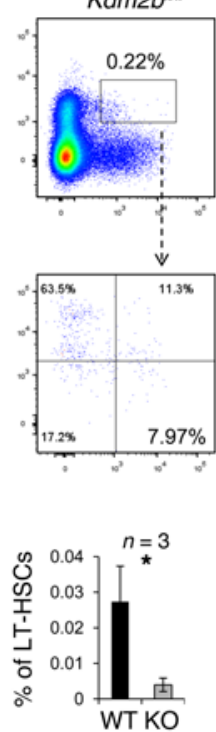

Vav1-Cre Kdm2b $b^{a m}$

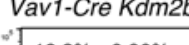

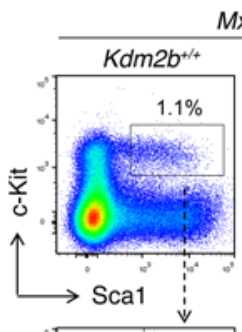
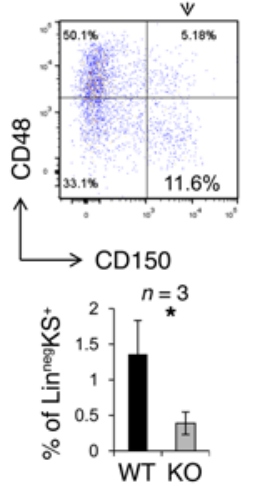

Gate B: Lin ${ }^{\text {neg KS }}$

$\%$ of $\mathrm{BM}$

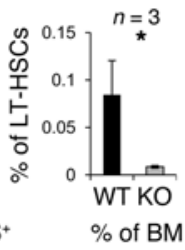

$0.12]_{\substack{* * \\ n=3}}^{n}$

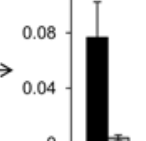

LMPPS

Gate C: Lin ${ }^{\text {neg Klow }} \mathrm{S}^{\text {low }} \quad \%$ of BM

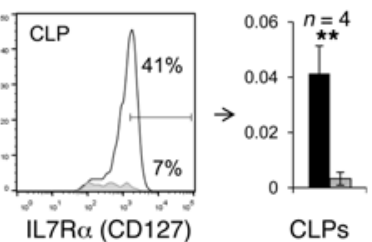

CLPs
C

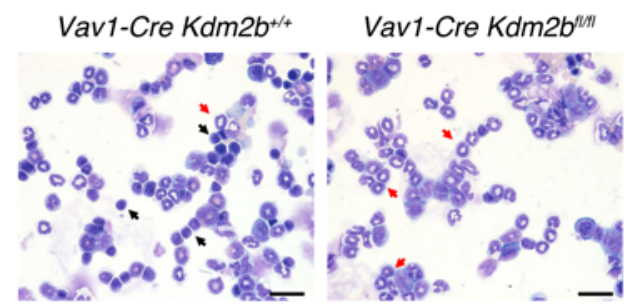

D

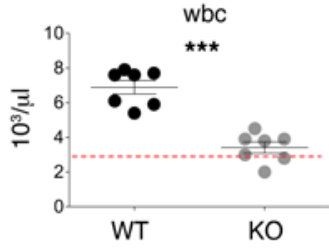

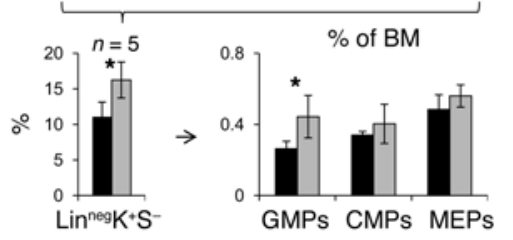

- Vav1-Cre Kdm2b+/4

ㅁ Vav1-Cre Kdm2bint

Figure 2. KDM2B is required for maintenance and lineage commitment of HSPCs. (A) Flow cytometric analysis of Vav1-Cre (left) and Mx1-Cre mice 2 months after plpC (right) showing Lin ${ }^{\text {nes }} \mathrm{KS}^{+}$and LT-HSC frequencies (\%) in BM of 10- to 15-week-old WT and Kdm2b-null mice. Bar graphs show mean \pm SEM. ${ }^{*} P<0.05 ;{ }^{* *} P<0.01$. (B) Composite analysis showing the frequency (\%) of lymphoid (gate B for LMPP and gate C for CLP) and myeloid (gate A for MEP, CMP, and GMP) progenitors. Bold red type denotes the gates, and black numbers denote the percentages of gated cells. Right: bar graphs show the contribution (\%) of LMPPs and CLPs in BM. Bottom: bar graphs depicting the percentages of Lin $^{\text {nes }} \mathrm{K}^{+} \mathrm{S}^{-}$cells and the frequency (\%) of GMP, CMP and MEP in BM. ${ }^{*} P<0.05$; ${ }^{*} P<0.01$. (C) May-Grünwald Giemsa-stained cytospin preparations of WT and $K d m 2 b$-null BM. Note the rarity of lymphocytes (black arrows) and the expansion of immature myeloid cells (red arrows) in Kdm2b-null BM. Scale bars: $10 \mu \mathrm{m}$. (D) Top: scatter plots of wbc counts. Red dotted line indicates minimum physiological range. Bar graphs show the absolute number of lymphocytes (LYM), monocytes (MONO), granulocytes (GRAN), platelets (PLT), and rbc. Bottom: representative flow cytometric analysis and cumulative scatter plot showing the frequency (\%) of T, B, and myeloid (M) cells in the peripheral blood. ${ }^{*} P<0.05$. NS, not significant.

expression of reverse tetracycline-controlled transcriptional activator (rtTA) and GFP occurred through removal of the "lox-stop-lox" cassette to allow hematopoietic-specific and doxycycline-inducible expression of WT or demethylase-deficient KDM2B that mimics the short isoform (Figure 3A, hereafter referred to as Tet-Kdm2b and Tet-Kdm2 $\left.b^{\lrcorner J m j c}\right)$. Supplemental Figure 2, G-I, shows mosaic expression of KDM2B in thymus and spleen. Ectopic expression of KDM2B caused a 2-fold expansion of $\mathrm{Lin}^{\text {neg }} \mathrm{KS}^{+}$, LMPPs, and CLPs in a JmjC domain-dependent manner, without altering the absolute number of LT-HSCs (Figure 3B). No significant changes were observed in the frequency of $\mathrm{Lin}^{\text {neg }} \mathrm{K}^{+} \mathrm{S}^{-}$cells, CMPs, MEPs, or GMPs. Consistently, hematopoietic progenitor colony-forming assays showed that KDM2B favored lymphoid and inhibited myeloid differentiation in a JmjC domain-dependent manner (Supplemental Figure 2J). Analy- 
A
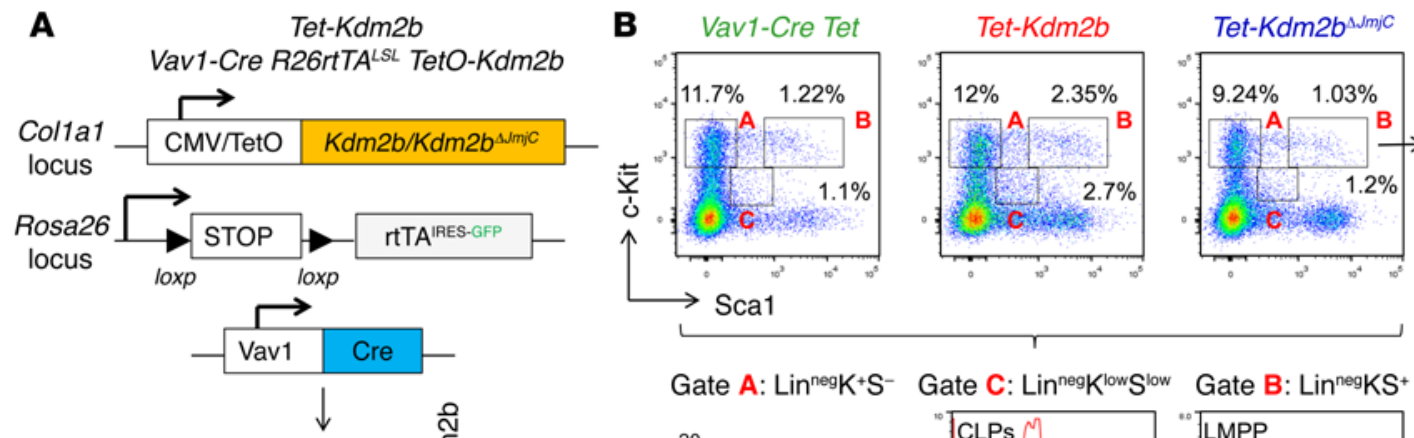

Gate A: Lin ${ }^{\text {neg }} \mathrm{K}+\mathrm{S}$

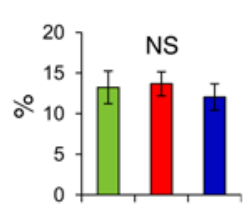

Gate C: Lin ${ }^{\text {neg Kow }}$ S $^{\text {low }}$

Gate B: Lin ${ }^{\text {neg KS }}+$
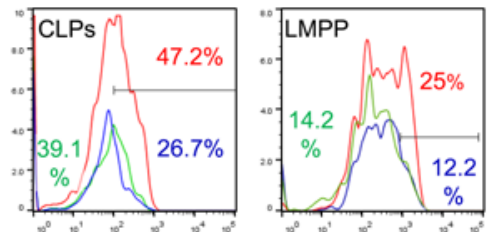

IL7R $\alpha$ (CD127)

$\mathrm{Lin}^{\mathrm{neg}} \mathrm{K}^{+} \mathrm{S}^{-}$

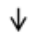

$n=2$ Actin GFP
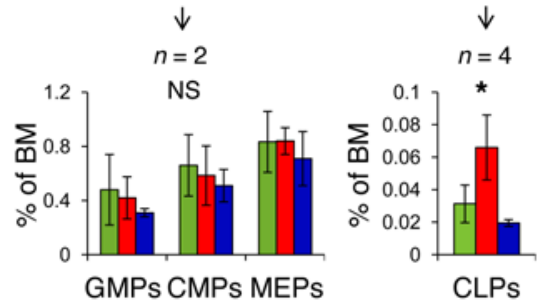

Flt3 (CD135)

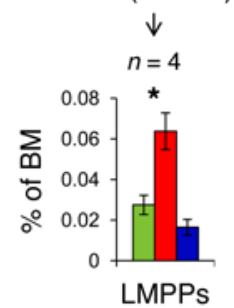

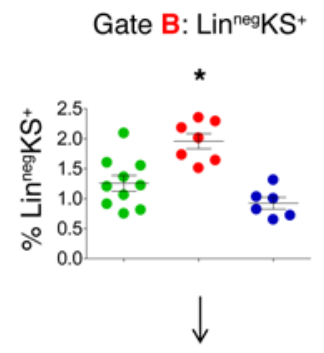

$\%$ of LT-HSCs in BM

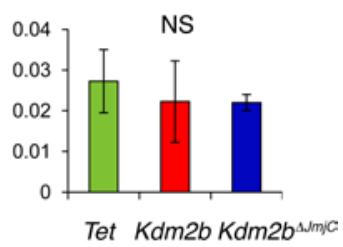

Tet $K d m 2 b K d m 2 b^{\Delta / m j c}$

$\square$ Tet

- Tet-Kdm2b

- Tet-Kdm2 $b^{\Delta\lrcorner m j c}$

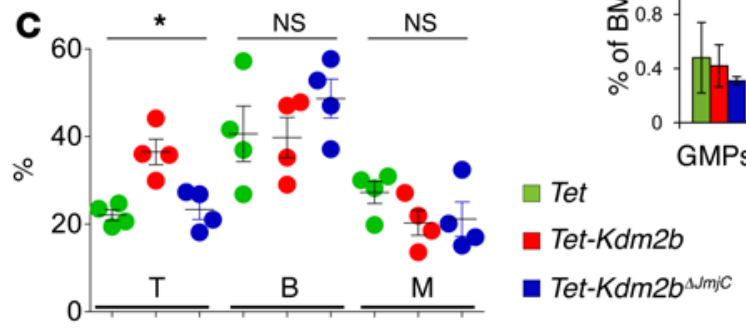

Figure 3. Ectopic expression of KDM2B expands lymphoid-primed progenitors in a Jumonji domain-dependent manner. (A) Schematic of doxycyclineinducible $K d m 2 b$ or $K d m 2 b^{4 / m j c}$ mice. Crosses with Vav1-Cre removed the "lox-stop-lox" cassette and allowed the expression of rtTA and GFP in the hematopoietic system, which was confirmed by flow cytometry. Bottom: Lin ${ }^{\text {neg }}$ BM cells were isolated from the indicated mice and cultured in the presence of doxycycline for 3 days. Ectopic expression of KDM2B was confirmed by Western blotting probing with an anti-MYC antibody that recognizes the exogenous MYC-tagged protein. (B) Composite analysis showing the frequency (\%) of lymphoid (gate B for LMPP and gate C for CLP) and myeloid (gate A for MEP, CMP, and GMP) progenitors in mice that were administered doxycycline for 9 to 12 weeks to express KDM2B or KDM2B ${ }^{\Delta / m i c}$. Bold red type denotes the gates, and black numbers denote the percentages of gated cells. Bar graphs show the contribution (\%) of GMPs, CMPs, MEPs, CLPs, LMPPs, and LT-HSCs in BM. ${ }^{*} P<0.05$, ANOVA. (C) Scatter plot showing the frequency (\%) of T, B, and myeloid cells in doxycycline-treated mice of the indicated genotypes. ${ }^{*} P<0.05$, ANOVA. NS, not significant.

sis of peripheral blood showed a significant expansion of $\mathrm{T}$ cells without affecting B and myeloid cells (Figure 3C). We conclude that, in our system, ectopic expression of KDM2B in hematopoietic progenitors favors T lymphocyte commitment in vivo.

Intrinsic role of $K d m 2 b$ in lineage commitment. We performed noncompetitive BM transplantations of CD 45.2 Vav1-Cre $\mathrm{Kdm}_{2} \mathrm{~b}^{+/+}$ or Vav1-Cre Kdm2 $b^{\text {fl/ }}$ donor cells into lethally irradiated CD 45.1 congenic recipients to determine whether the role of KDM2B in hematopoiesis is cell intrinsic (Supplemental Figure 3A). After confirming successful reconstitution, we analyzed the peripheral blood of recipient animals over time. Interestingly, we found that mice who received $K d m 2 b$-null BM showed a reconstitution defect over time (Figure 4A), manifested in hypocellularity, low white blood cell (wbc) counts, and trilineage cytopenia (Figure 4B and Supplemental Figure 3B). To rule out the possibility that lack of KDM2B affected cell migration, we performed competitive BM transplantations, transplanting equal numbers of donor CD $45.2 \mathrm{Mx1}-\mathrm{Cre} \mathrm{Kdm}_{2} \mathrm{~b}^{+/+}$or Mx1-Cre Kdm2 $b^{A / f l}$ and competitor CD45.1 cells into lethally irradiated CD45.1 recipients (Figure 4C and Supplemental Figure 3C). After documenting successful engraftment, $p I p C$ was administered to excise $K d m 2 b$. Mice transplanted with $K d m 2 b$-null BM displayed a decline in the level of CD45.2 donor cells similar to that seen in the Vav1-Cre experiment, while mice transplanted with $\mathrm{M} x \mathrm{x}$-Cre $\mathrm{Kdm} 2 \mathrm{~b}^{+/+} \mathrm{BM}$ showed the opposite trend (ratio of CD45.2/CD45.1 cells in Figure 4C). KDM2B-deficient CD45.2 donor cells showed reduced reconstitution of $\mathrm{B}$ and $\mathrm{T}$ lymphocytes in the peripheral blood, suggesting an intrinsic role in lymphopoiesis (Figure 4D).

$K D M 2 B$ regulates transcriptional programs involved in cell identity and cell cycle. To uncover the molecular pathways regulated by KDM2B, RNA sequencing (RNA-seq) was performed on $K d m 2 b$ null and WT $\mathrm{Lin}^{\text {neg }} \mathrm{KS}^{+}$hematopoietic progenitors because we failed to isolate sufficient numbers of LT-HSCs from $K d m 2 b$-KO mice (Figure 2A). Bioinformatics analysis revealed 1,032 differentially expressed genes $(P<0.05$ and fold change $>1.5) ; 251$ genes were upregulated and 781 genes downregulated upon $K d m 2 b$ deletion (Figure 5A and Supplemental Table 1). Interestingly, several bona fide polycomb targets (33), including Hox, Wnt, and $F z d$, were upregulated, indicative of active canonical and noncanonical Wnt signaling. In contrast, transcription factors that regulate hematopoietic development (Gata1, Hes1, Tcf7, and Gfi1b), several lymphocyte- 


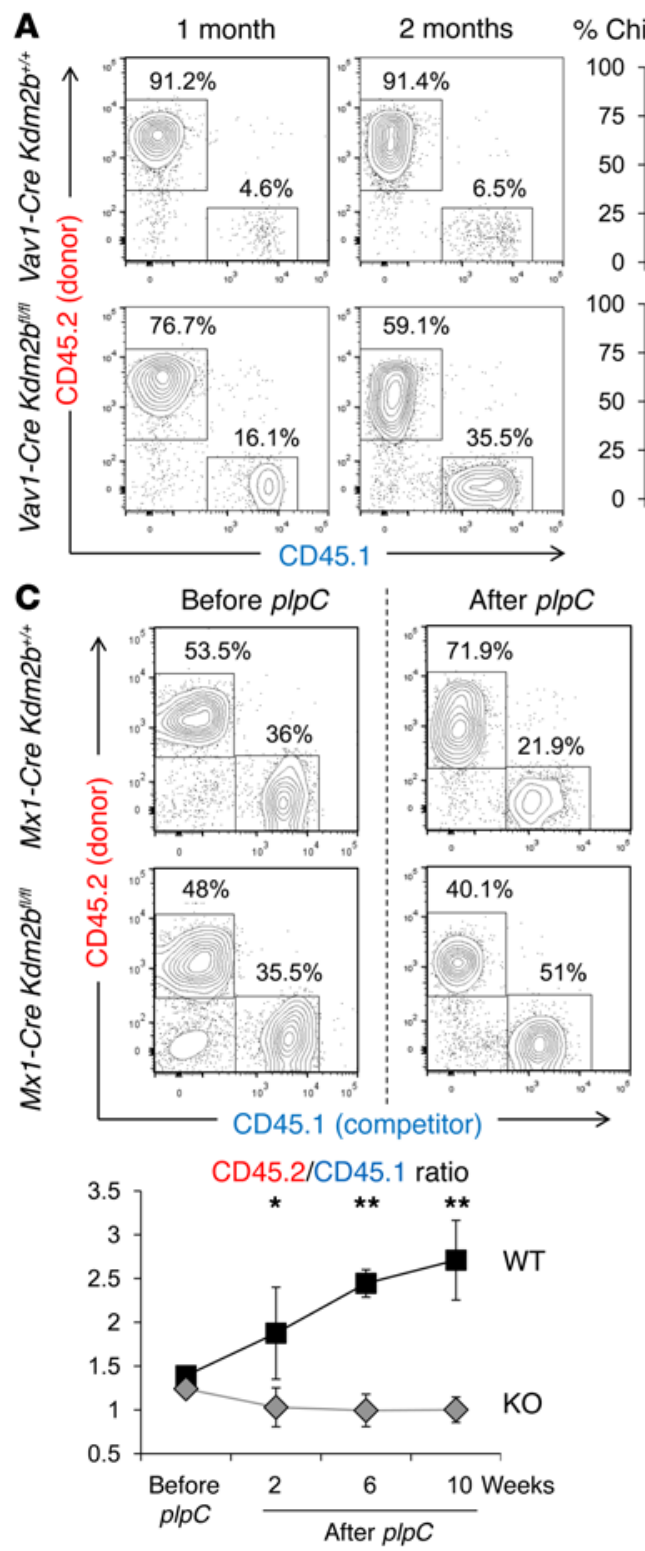

B
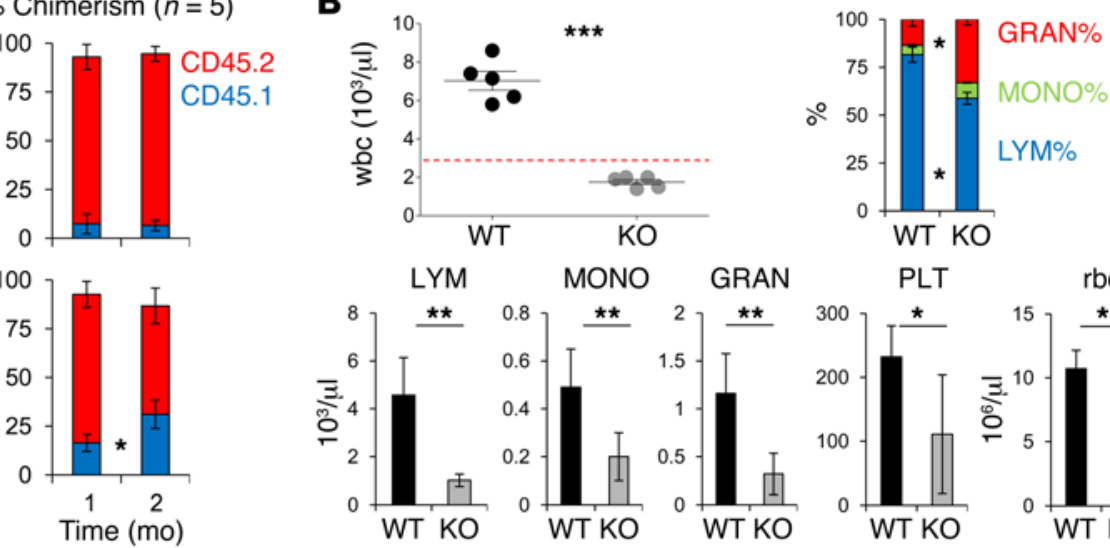

GRAN
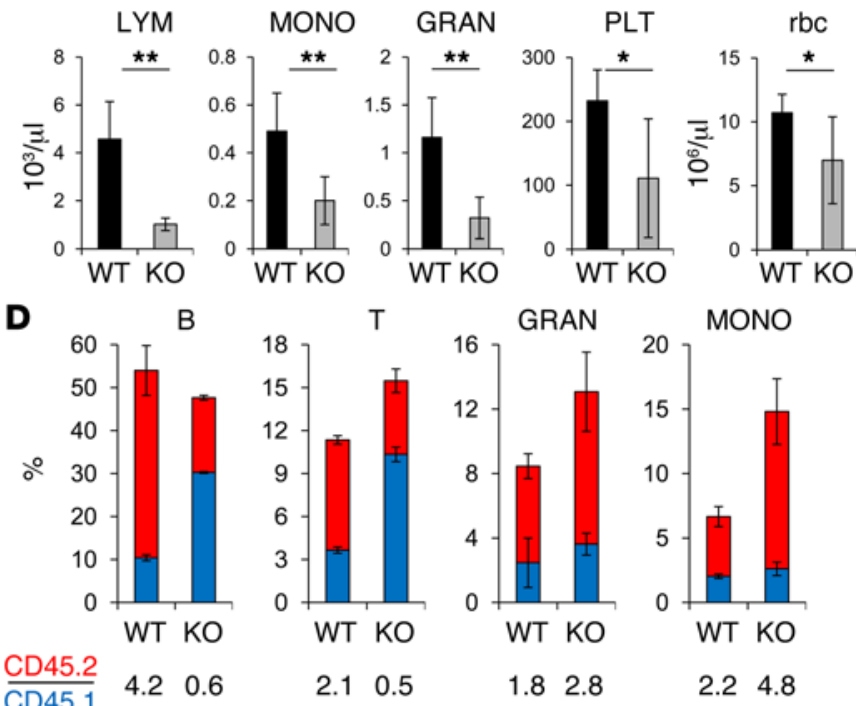

Figure 4. Intrinsic role of KDM2B in lineage commitment. (A) $1 \times 10^{6}$ CD45.2 WT or $K d m 2 b$-null BM cells were injected into lethally irradiated CD45.1 recipients. Left: representative flow cytometric analysis showing the frequency (\%) of CD45.2 and CD45.1 cells 1 and 2 months after transplantation. Right: cumulative bar graph from 5 independent transplantations. ${ }^{*} P<0.01$ for CD45.1 cells. (B) Upper left: scatter plot of wbc in transplanted mice. Red dotted line indicates minimum physiological range. Upper right: stacked bar graph depicts the contribution (\%) of lymphocytes, monocytes, and granulocytes. Bottom: bar graphs show the absolute number of lymphocytes, monocytes, granulocytes, platelets, and rbc. ${ }^{*} P<0.05 ;{ }^{* *} P<0.01$; ${ }^{* *} P<0.001$. (C) $1 \times 10^{6} \mathrm{WT}$ or $K d m 2 b^{f / f f}$ CD45.2 BM cells were injected in a 1:1 ratio with CD45.1 competitor cells. Left: flow cytometric analysis shows the frequency (\%) of CD45.2 and CD45.1 cells before and 10 weeks after plpC administration. Right: line graph showing the ratio of CD45.2/CD45.1 cells in recipients over time. $n=4$. ${ }^{*} P<0.05$; ${ }^{* *} P<0.01$. (D) Stacked bar graphs showing the contribution (\%) of CD45.2 and CD45.1 cells in B and T cells, granulocytes, and monocytes after plpC administration. Bottom: ratio of CD45.2 to CD45.1 cells. specific antigens ( $L y 6 i, L y 6 c 1$, and $L y 6 g 6 f)$, and a regulator of the hematopoietic niche (Angpt1) (34) were downregulated. Notably, several mediators of the interferon response were downregulated, such as Irf4 and Irf7 transcription factors, which are also important for lymphoid development and maturation of T cells $(35,36)$. qRTPCR in Lin $^{\text {neg }} \mathrm{KS}^{+}$progenitors confirmed the RNA-seq results (Figure 5A). Similarly, analysis of $\operatorname{Lin}^{\text {neg }}$ cells revealed downregulation of several hematopoietic cell-surface receptors (Flt-3 and Il7ra) and transcription factors (Tal1, Pax5, Ikzf1, Ikzf2, and Hes1) that regulate lymphoid commitment; conversely, mediators of Wnt signaling were upregulated (Figure 5B). Ectopic expression of KDM2B confirmed the reciprocal regulation of those genes (Figure 5B). Furthermore, flow cytometric analysis showed a 3-fold decrease in the percentage of cells expressing master lymphoid regulators IKAROS and PAX5 (Figure 5C). Ingenuity Pathway Analysis (IPA; http://www.ingenuity.com/products/ipa) (Figure 5D) and Gene Set Enrichment Analysis (GSEA; http://software.broadinstitute.org/ gsea/index.jsp) (Figure 5E and Supplemental Figure 4, A and B) revealed that loss of $K d m 2 b$ caused gene-expression changes that can be categorized into 3 groups: (a) pathways that regulate lineage choice and cell identity, such as Notch and Wnt, (b) cytokine/interferon signaling, and (c) activation of cell cycle. Accordingly, flow cytometric analysis of the cell-cycle status of $\mathrm{Lin}^{\text {neg }} \mathrm{KS}^{+}$progenitors confirmed that loss of KDM2B significantly increased the number of cells in the $\mathrm{S} / \mathrm{G}_{2} / \mathrm{M}$ phases of the cell cycle (Figure $5 \mathrm{~F}$ ).

KDM2B is required for the maintenance of human leukemiccell lines. We interrogated $K D M 2 B$ expression in more than 1,000 human cancer cell lines through the Cancer Cell Line Encyclopedia (CCLE) (ref. 37; http://www.broadinstitute.org/ccle/home) and found that cell lines established from hematopoietic malignancies had higher expression of KDM2B compared with epithelial cancers (not shown), particularly in acute leukemias and non-Hodgkin's B cell lymphomas compared with multiple myeloma (MM) and Hodgkin's lymphoma (HL) (Figure 6A). Western blotting confirmed the broad expression 
A

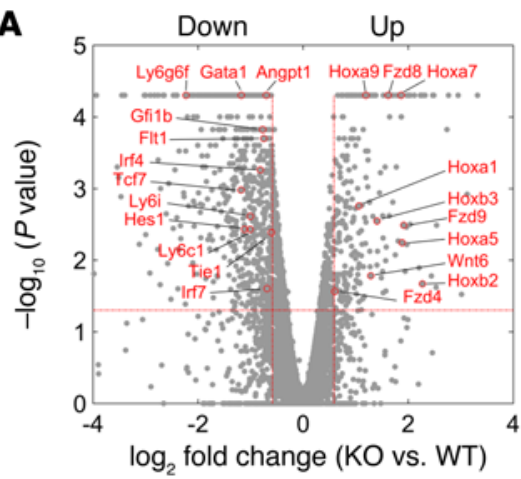

B

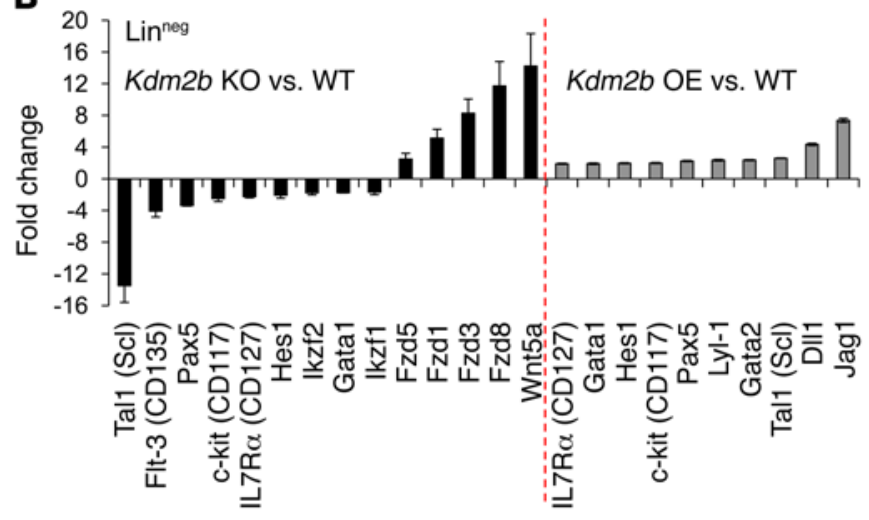

E

GSEA Leading edge analysis

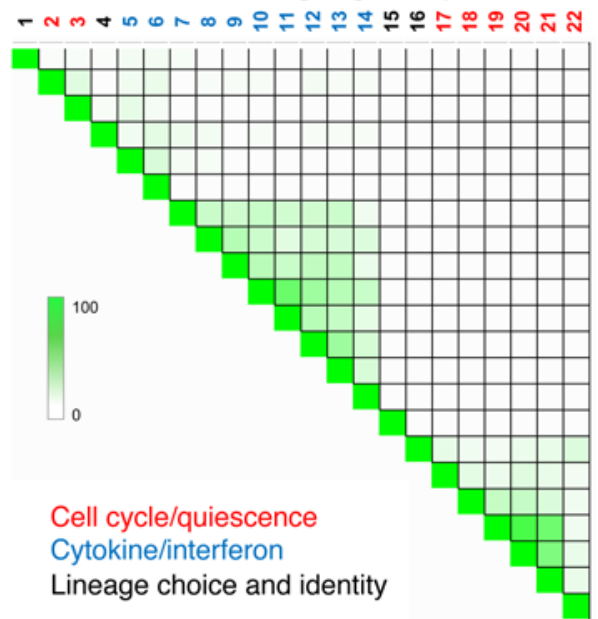

4. VILIMAS_NOTCH1_TARGETS_UP

5. SCHOEN NFKB SIGNALING

7. HECKER_IFNB1_TARGETS

9.MOSERLE_IFNA_RESPONSE

16. FEVR_CTNNB1_TARGETS_DN

17. ISHIDA_E2F_TARGETS

20. REACTOME_G1_S_TRANSITION

22. MANOLO_HYPOXIA_DN

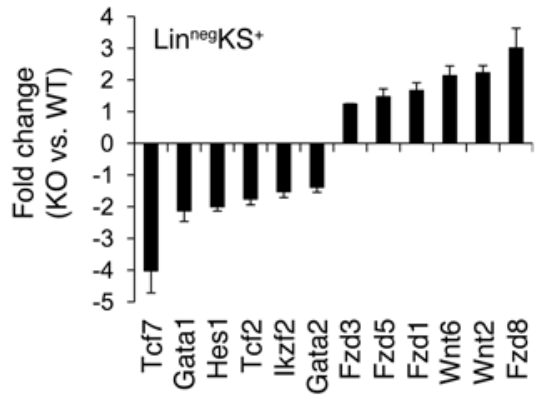

C

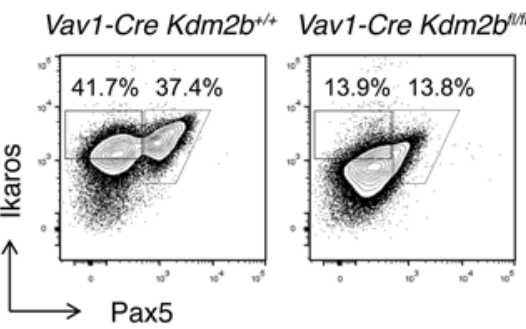

D

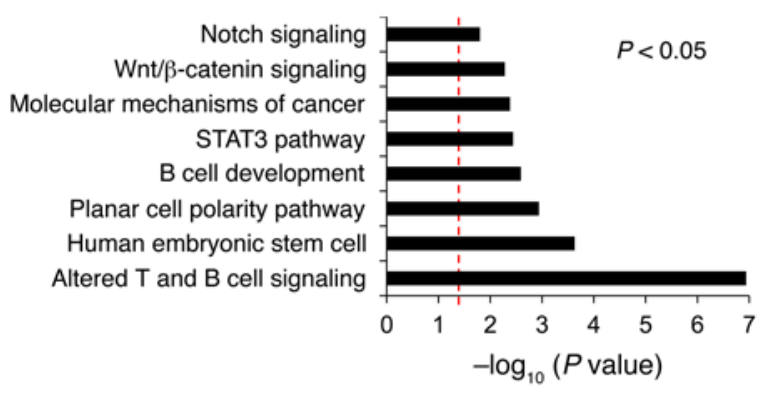

$\mathbf{F}$

KEGG_HEMATOPOIETIC_CELL_LINEAGE

2. NORMAL_QUIESCENT_VS_NORMAL_DIVIDING_UP 3. CML_QUIESCENT_VS_NORMAL_DIVIDING_UP

6. TIAN_TNF_SIGNALING_VIA_NFKB

8. BOSCO INTERFERON INDUCED_ANTIVIRAL MODULE

10. REACTOME INTERFERON ALPHA BETA SIGNALING

11. ZHANG_INTERFERON_RESPONSE

12. BROWNE_INTERFERON_RESPONSIVE_GENES

13. RADAEVA_RESPONSE_TO_IFNA1_UP

14. REACTOME INTERFERON GAMMA SIGNALING

15. PID_WNT_SIGNALING_PATHWAY

18. REACTOME G2_M_CHECKPOINTS

19. REACTOME M G1 TRANSITION

21. REACTOME_MITOTIC_M_M_G1_PHASES

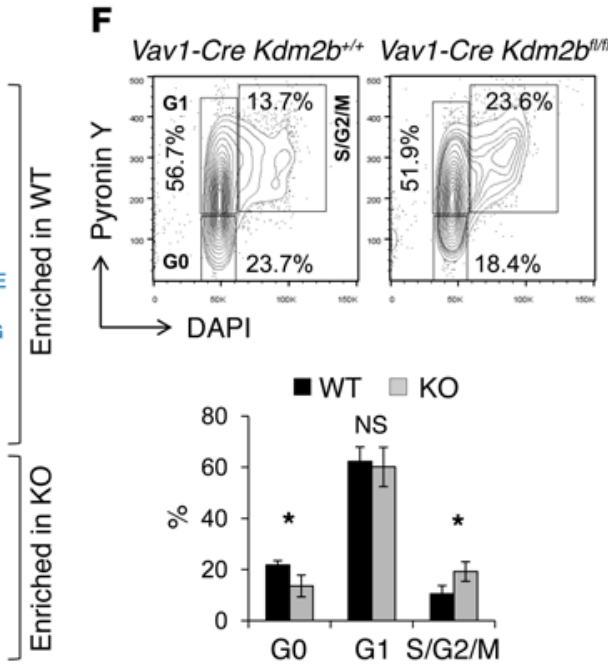

Figure 5. KDM2B regulates cell fate and cycling of HSPCs. (A) Volcano plot shows the differentially expressed genes $(P<0.05$ and fold difference $>1.5)$ in $K d m 2 b$-null Lin ${ }^{\text {neg }} \mathrm{KS} S^{+}$progenitors compared with WT $(n=2)$. Red circles highlight the position of the indicated transcripts. Right: bar graph (mean \pm SD) shows fold difference of the indicated transcripts in Lin ${ }^{\text {neg }}$ KS+ by qRT-PCR. (B) Bar graph (mean \pm SD) shows the fold difference determined by qRT-PCR in the expression of the indicated transcripts in Kdm2b-null (black bars) and KDM2B-overexpressing (OE, gray bars) Lin ${ }^{\text {neg }}$ cells compared with WT controls. (C) Intracellular flow cytometric analysis of IKAROS and PAX5 transcription factors in WT and $K d m 2 b$-null Lin ${ }^{\text {neg }}$ BM. (D) IPA of the differentially expressed genes identified in $\mathbf{A}$. The $x$ axis (log scale) corresponds to the binomial raw $P$ values. (E) GSEA leading edge analysis identified 3 distinct modules regulated by KDM2B in Lin ${ }^{\text {neg }} \mathrm{KS}^{+}$cells. Color bar shows the overlap (\%) between the individual gene sets. (F) Cell-cycle analysis of Lin ${ }^{\text {neg }} \mathrm{KS}^{+}$cells. $n=2$. ${ }^{*} P<0.05$.

of KDM2B in leukemias, but not in MM cell lines (Supplemental Figure $5 \mathrm{~A}$ and data not shown). When screened for possible copy number changes, only a few cell lines (classified as MM and AML) exhibited loss of heterozygosity (Supplemental Figure 5B). Additional analysis through The Cancer Genome Atlas (TCGA; http:// cancergenome.nih.gov/) and other publicly available databases did not identify structural changes (mutations, truncations, and/or chromosomal translocations) involving the KDM2B locus (not shown).
Leukemias are heterogeneous diseases that commonly harbor mutations in genes that regulate lineage commitment (such as PAX5, IKZF1, and FLT3, among others) and chromosomal translocations that lead to the formation of oncogenic fusion proteins. Given that KDM2B is highly expressed in leukemias, we analyzed the RNA-seq profiles of 81 leukemias by means of $K D M 2 B$ expression, leukemia classification, and cytogenetic abnormalities (Supplemental Figure 5C). Besides confirming that KDM2B is preferentially 

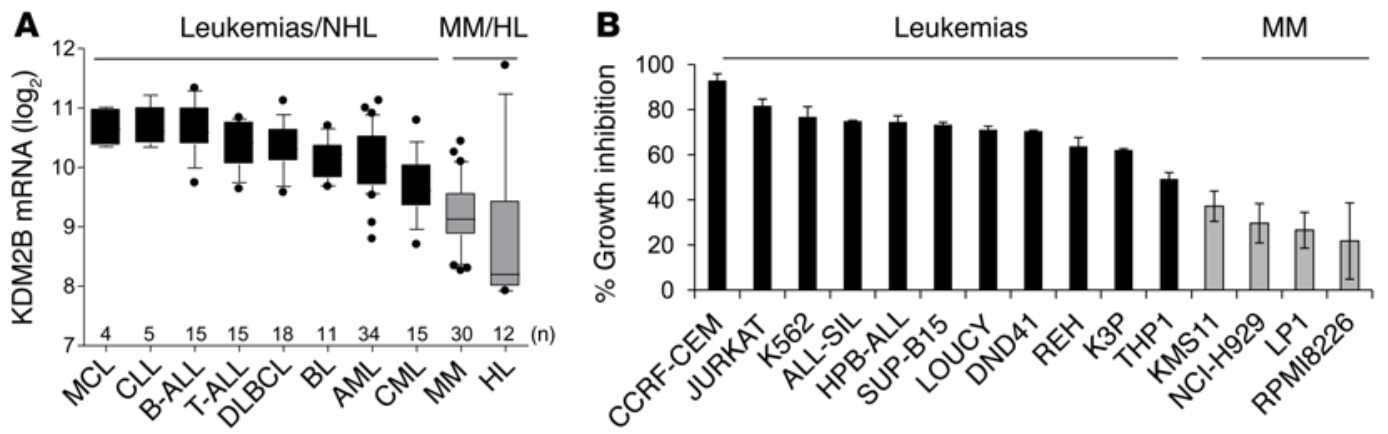
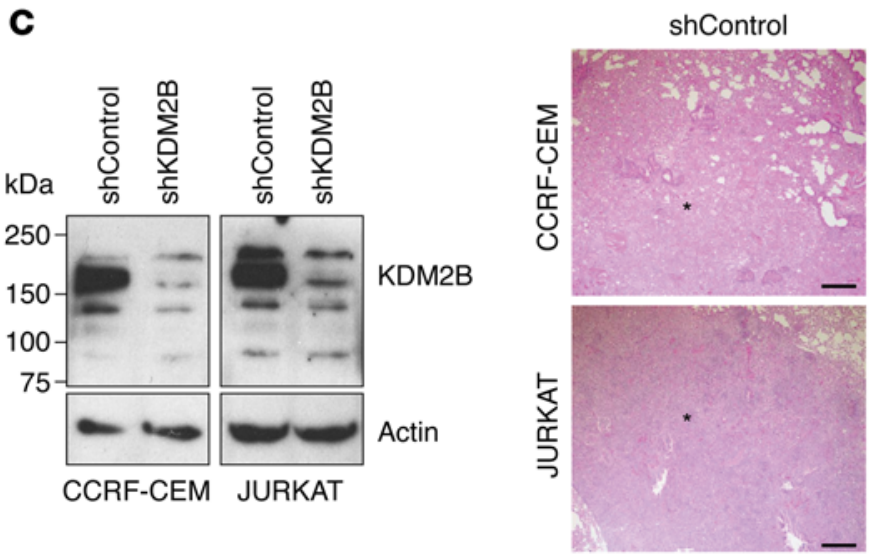

D

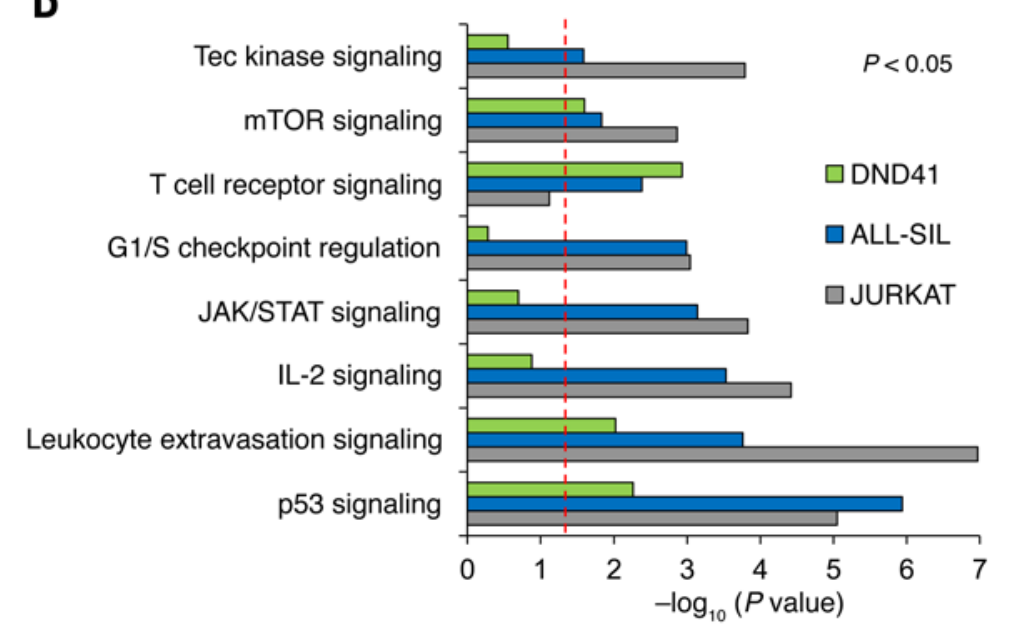

shKDM2B

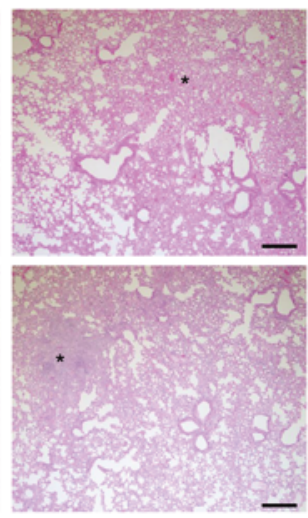

$\mathbf{E}$

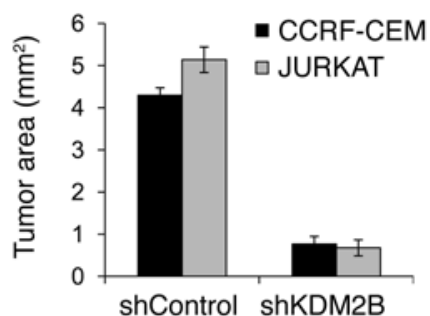

shControl shKDM2B

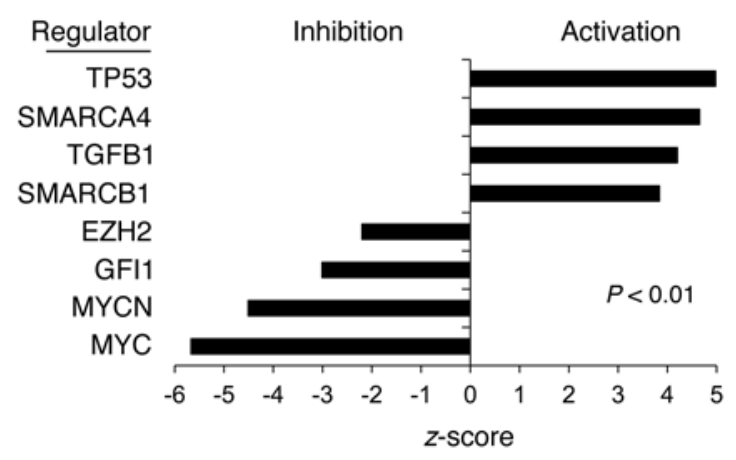

Figure 6. Prooncogenic role of KDM2B in lymphoid malignancies. (A) Meta-analysis of KDM2B expression in 159 human cell lines established from leukemias, lymphomas, and MM obtained from CCLE. MCL, mantle cell lymphoma; DLBCL, diffuse large B cell lymphoma; BL, Burkitt's lymphoma; NHL, non-Hodgkin's lymphomas. (B) Percentage growth inhibition of B-ALL (SUP-B15 and REH), T-ALL (CCRF-CEM, JURKAT, ALL-SIL, HPB-ALL, LOUCY, DND41, and K3P), CML (K562), AML (THP1), and MM cell lines 7 days after KD of KDM2B. Results are mean \pm SEM. (C) The indicated cell lines were infected with lentiviruses to KD KDM2B. Left: Western blot shows successful KD of the endogenous protein. Middle and right: $1 \times 10^{6}$ of those cells were injected in NSC mice via tail vein ( $n=3$ per group). After 3 months, mice were euthanized for histological analysis. H\&E staining of lung sections shows reduced metastasis in mice that received cells treated with shKDM2B. Bar graph shows the average area of lung nodules. Asterisk indicates metastatic nodule. Scale bars: $200 \mu \mathrm{m}$. (D) Indicated cell lines were infected with lentiviruses to KD KDM2B. RNA was isolated and the gene-expression profile was obtained using the PrimeView microarray (Affymetrix). Bar graph shows IPA of the differentially expressed genes $(P<0.05$ and fold change $>1.5)$. The $x$ axis (log scale) corresponds to the binomial raw $P$ values. (E) Ingenuity regulator analysis (see Methods) in JURKAT cells treated with shKDM2B. Regulators with $z$ scores greater than 2 and $P$ overlap values of less than 0.01 are shown.

expressed in acute lymphoblastic leukemia (ALL) versus AML, we found no correlation between KDM2B expression and cytogenetic abnormalities, except in Epstein-Barr virus-positive B cell chronic lymphocytic leukemia (CLL)/small lymphocytic lymphoma (SLL) cell lines, which had the highest expression (Supplemental Figure $5 \mathrm{C})$. Thus, we knocked down KDM2B using shRNA in several cell lines that contain a spectrum of cytogenetic abnormalities, representing ALL, chronic myeloid leukemia (CML), AML, and MM (Figure 6B, Supplemental Figure 5D, and data not shown). We found that KDM2B-targeted shRNA-treated (shKDM2B-treated) leukemia, but not MM, cell lines ceased to proliferate (Figure 6B), consistent with the KDM2B expression pattern (Figure 6A). Furthermore, 
shKDM2B-treated cells had reduced metastatic potential upon injection into NOD-scid Il2rgnull (NSG) mice (Figure 6C). Geneexpression profiling in T-ALL cell lines, such as JURKAT, ALLSIL, and DND41, revealed that shKDM2B caused alterations of p53-dependent pathways, cell-cycle regulation, and JAK/STAT and mTOR signaling as well as pathways involved in differentiation, survival, and proliferation of T cells (Figure 6D). Furthermore, IPA prediction of regulators that could mechanistically explain the observed changes in gene expression indicated downregulation of MYC- and EZH2-dependent transcriptional programs after treatment with shKDM2B. In contrast, tumor-suppressor pathways downstream of p53, TGFB1 receptor, SMARCA4/BRG1, and SMARCB1/SNF5 were upregulated (Figure 6E). Of note, the latter are components of the SWI/SNF chromatin remodeling complex, which antagonizes EZH2/PRC2 (38). Overall, we infer that KDM2B is required for the maintenance of human leukemia cell lines through regulation of cell fate and lymphocyte-specific signaling pathways.

$K D M 2 B$ restrains $K R A S^{G 12 D}$-driven myeloid transformation. Although KDM2B rarely exhibits structural or copy number changes (Supplemental Figure 5B), its partners - such as BCOR and components of PRC2 (39) - are frequently mutated in MDS, AML, juvenile myelomonocytic leukemia (JMML), and a subset of adult T-ALL $(14,15,40,41)$. Given that $K d m 2 b$ ablation compromised lymphopoiesis and skewed differentiation toward the myeloid lineage (Figure 2), we hypothesized that this may accelerate AML in the context of the appropriate oncogenic driver. In support of this hypothesis, meta-analysis of the TCGA AML (LAML) cohort (15, 42) revealed that low expression of KDM2B and EZH2 significantly correlated with aggressive disease (Figure 7A). For our studies, we employed a mouse model of AML driven by $\operatorname{Kras}^{G 12 D}$ (43), since RAS/ERK signaling is frequently activated in AML through gainof-function mutations in either KRAS, NRAS, or FLT3 (15). In the context of $K r a s^{G 12 D}$, Vav1-Cre Kdm2 $b^{f / f l}$ mice developed aggressive acute transformation and succumbed to the disease before 6 weeks of age (Figure 7B). Analysis of the peripheral blood revealed high wbc counts and the presence of circulating myeloblasts (Figure 7, $\mathrm{C}$ and $\mathrm{D}$ ). Immunophenotypic analysis of $\mathrm{BM}$ showed a precipitous drop of $\mathrm{Lin}^{\text {neg }} \mathrm{KS}^{+}$and $\mathrm{Lin}^{\text {neg }} \mathrm{K}^{\mathrm{lo}} \mathrm{S}^{\text {lo }}$ lymphoid progenitor populations as well as circulating B and T cells (Figure 7C and Supplemental Figure 6A). This was accompanied by a massive expansion of GMPs in $K d m 2 b$-null animals (Supplemental Figure 6A). Autopsy revealed splenomegaly and infiltration of liver, lung, and spleen (Supplemental Figure 6, B and C). Gene-expression profiling of leukemic cells, followed by IPA (Figure 7E) and GSEA leading edge (Supplemental Figure 6D) analyses, revealed that ablation of $K d m 2 b$ activated cell cycle, attenuated the activity of interferon and lymphoid-specific transcription factors, and derepressed PRC2-bound genes (Figure 7E and Supplemental Figure 6D). Overall, these findings provide a causal link between loss of KDM2B and myeloid transformation, consistent with the tumor-suppressor role of PRC2 in AML.

Next, we performed the reciprocal experiment by ectopically expressing KDM2B in the context of KRAS. Remarkably, these mice restored wbc to almost normal levels and expanded lymphocytes in a JmjC domain-dependent manner (Figure 8A and Supplemental Figure 6E), particularly CD3e $\mathrm{e}^{+} \mathrm{T}$ cells (Supplemental Figure $6 \mathrm{~F}$ ). Autopsy revealed reduced infiltration of peripheral tissues (Supplemental Figure 6G), which correlated with the prolonged survival of mice expressing WT, but not demethylation-deficient, KDM2B (Figure 8B). Gene-expression profiling of leukemic cells, followed by IPA (Figure 8C) and GSEA (Supplemental Figure 6H) leading edge analyses, indicated that overexpression of KDM2B potently activated transcriptional programs linked to interferon signaling (Irf and Stat transcription factors) and inhibited PRC2 antagonists (38) Hoxa1O and Smarca4/Brg1 (Figure 8C and Supplemental Figure $6 \mathrm{H}$ ). We also noticed that overexpression of KDM2B induced gene sets that are enriched in both embryonic stem cells and undifferentiated cancers, suggesting a block in KRAS-driven myeloid commitment. c-MYC-dependent gene signatures, frequently observed in lymphoid leukemias and lymphomas, were also upregulated (Supplemental Figure 6H). In conclusion, ectopic expression of KDM2B reverses the fate of KRAS-driven leukemias.

KDM2B cooperates with $P c G$ and $\operatorname{Tr} x G$ complexes to regulate cell fate decisions. To comprehensively identify the direct targets of KDM2B, we employed ChIP followed by high-throughput DNA sequencing (ChIP-seq) to map the genome-wide binding sites of the endogenous protein in B-ALL (REH, SUP-B15), T-ALL (JURKAT, HPB-ALL, ALL-SIL, DND41), and CML (K562). Peak calling revealed multiple genomic loci bound by KDM2B $\left(P<10^{-5}\right.$, Supplemental Figure 7A and Supplemental Table 2) preferentially enriched at gene promoters (Supplemental Figure 7B). KDM2B binds a core of 780 genes in all cell lines as well as distinct, cell-type-specific gene sets (Figure 9A). IPA of the core genes showed enrichment in cancer, pluripotency networks, cell fate, lineage commitment (NOTCH and WNT), and lymphocyte/cytokine signaling (Figure 9B). Analysis in individual cell lines further emphasized the significance of developmental pathways and revealed a preferential enrichment of mTOR, FLT3, and $\mathrm{T}$ cell receptor signaling in T-ALL, whereas noncanonical WNT (planar cell polarity) signaling was enriched in B-ALL and CML (Figure 9C). To further confirm this finding, we used GREAT (44) to interpret the binding pattern of KDM2B in the context of previously published profiles for transcription factors and epigenetic regulators contained in the Molecular Signatures Database (http://www. broadinstitute.org/gsea/msigdb). Interestingly, in CML (K562) and, to a lesser extent, in B-ALL - KDM2B-bound genes showed a preferential overlap with PRC2, whereas in T-ALL, which frequently harbors constitutive NOTCH activation, they preferentially overlapped with c-MYC (Supplemental Figure 7C). Furthermore, comparison of the genome-wide binding profile of NOTCH1 in HPB-ALL (45) revealed that approximately $52 \%$ of its targets were cobound by KDM2B (Supplemental Figure 7D); IPA indicated enrichment of pathways that regulate $\mathrm{T}$ cell homeostasis and $\mathrm{c}-\mathrm{MYC}$ signaling (Supplemental Figure 7D). Integration of gene-expression changes with ChIP-seq revealed that KDM2B functions as a transcriptional activator in JURKAT cells through association with c-MYC/TrxG, whereas in $\mathrm{K} 562$, it functions as a repressor through association with PRC2 (Supplemental Figure 7E). Accordingly, ChIP of selected KDM2B targets in shKDM2B-treated JURKAT cells showed that downregulated genes lost H3K4me3 and lacked H3K27me3 and H3K36me2, consistent with TrxG association, whereas upregulated genes lost H3K27me3 and gained H3K4me3 and H3K36me2, consistent with PcG association (Supplemental Figure 7F).

Large-scale ChIP-seq studies have indicated that epigenetic regulators and transcription factors are organized into distinct modules that can independently cobind common target genes - 
A

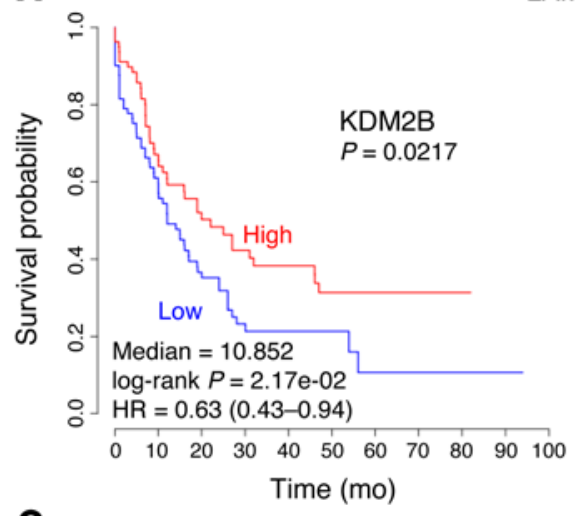

LAML

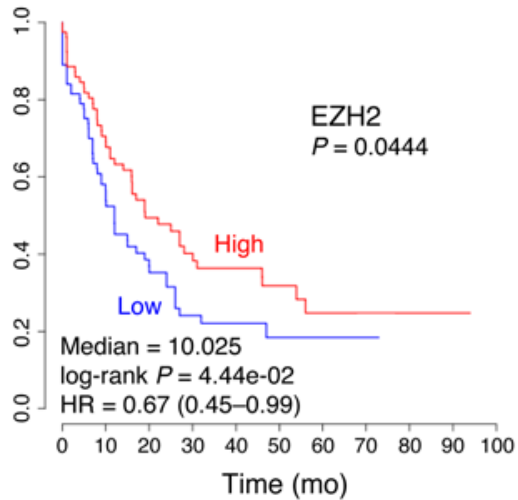

B

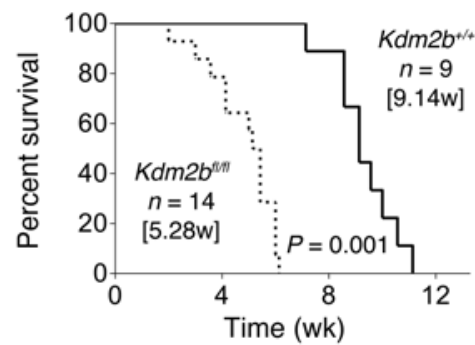

C
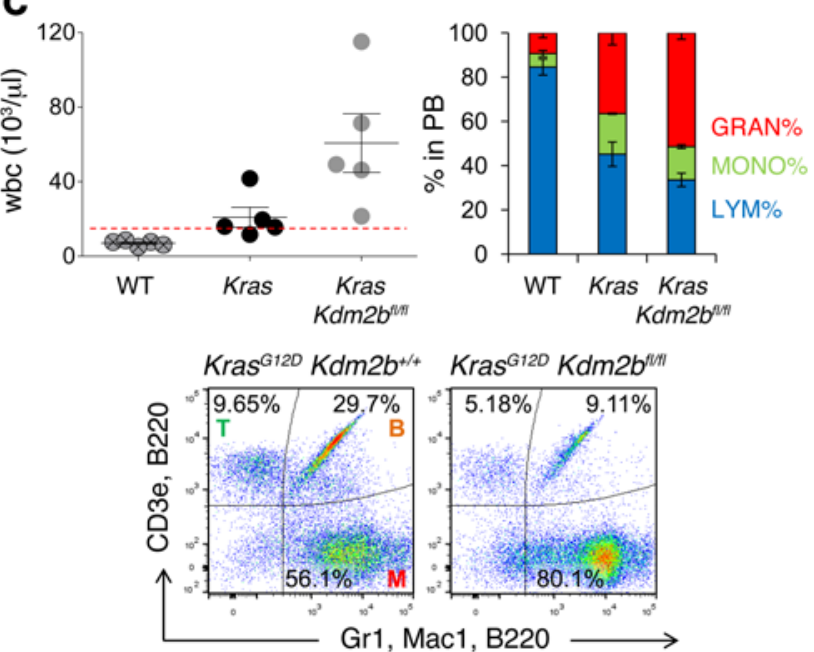

E Pathway

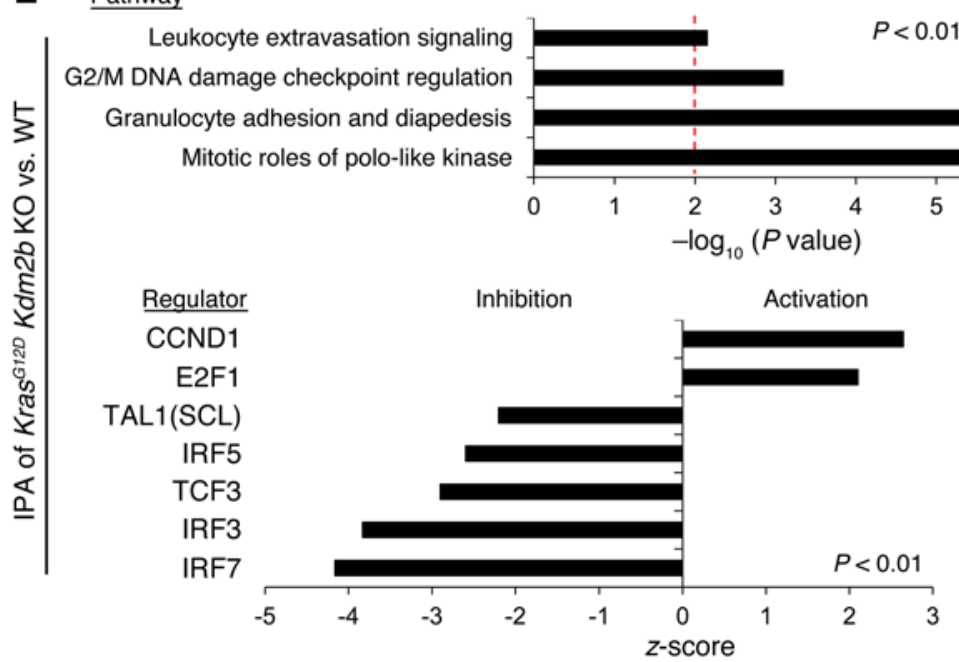

Figure 7. Ablation of $\boldsymbol{K} \boldsymbol{d} \mathbf{m} \mathbf{2} \boldsymbol{b}$ accelerates KRAS-driven myeloid transformation. (A) Kaplan-Meier plots show the survival of AML patients in the TCGA LAML cohort that express low and high levels of KDM2B (left) and EZH2 (right). Survival analysis was performed with the PRECOC profiles software. (B) Kaplan-Meier plot showing the survival of Vav1-Cre Kras ${ }^{G 12 D} \mathrm{Kdm} \mathrm{b}^{+/+}$(solid line) and Vav1-Cre Kras ${ }^{\mathrm{G} 120} \mathrm{Kdm} 2 \mathrm{~b}^{\mathrm{fl} / f l}$ (dotted line) mice. Median survival in brackets. (C) Scatter plot of wbc and stacked bar graph of percentage contribution of different lineages. $P<0.05$, ANOVA. Red dotted line indicates the maximum physiological range. Bottom: flow cytometry analysis showing the frequency (\%) of T, B, and myeloid cells in the peripheral blood. (D) May-Grünwald Giemsa staining of blood smears and BM cytospin preparations from 3- and 6-week-old mice. Black arrows, lymphocytes; red arrows, immature granulocytes; orange arrows, myeloblasts. Scale bars: $10 \mu \mathrm{m}$. (E) RNA was isolated from Lin ${ }^{\text {nes } V a v 1-C r e ~ K r a s}{ }^{6120} K d m 2 b^{+/+}$and $K d m 2 b^{f / f l}$ cells ( $n=2$ mice for each genotype), and gene-expression profiles were obtained with the MoGene 2.0 ST whole transcript (exon) microarray (Affymetrix). Bar graph shows IPA of the differentially expressed genes $(P<0.05$ and fold change $>1.4)$. The $x$ axis (log scale) corresponds to the binomial raw $P$ values. Bottom: ingenuity regulator analysis (see Methods) in Kdm2b-null KRAS-driven leukemias. Regulators with $z$ scores greater than 2 and $P$ overlap values of less than 0.01 are shown. 

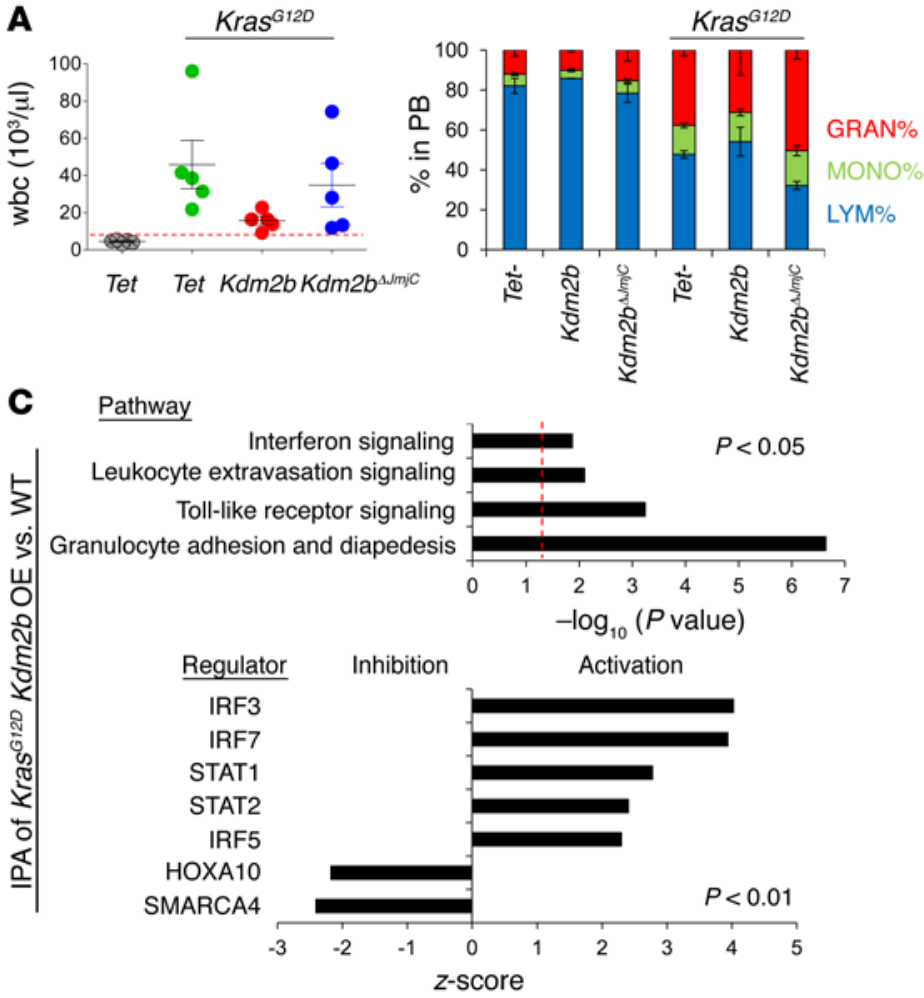

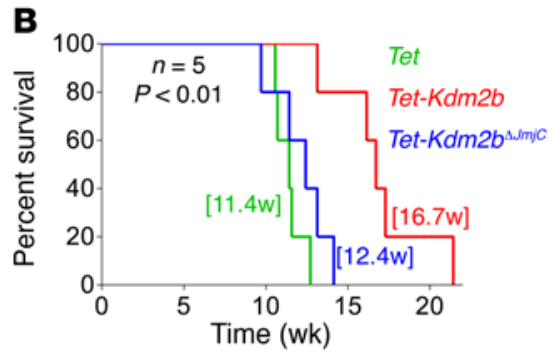

Figure 8. Ectopic expression of KDM2B antagonizes KRAS-driven myeloid transformation. (A) Left: scatter plot of wbc in Vav1-Cre Kras ${ }^{G 12 D}$ Tet-Kdm2b and Tet-Kdm2 $b^{4 / m i c}$ mice. $P<0.05$, ANOVA. Red dotted line indicates the maximum physiological range. Right: stacked bar graph shows the contribution (\%) of granulocytes, monocytes, and lymphocytes after doxycycline administration. (B) Kaplan-Meier plot showing the survival of Vav1-Cre Kras ${ }^{\mathrm{C} 120}$ Tet- $^{-}$

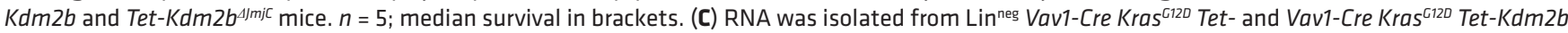
cells ( $n=2$ mice), and gene-expression profiles were obtained with the MoGene 2.0 ST whole transcript (exon) microarray (Affymetrix). Bar graph shows IPA of the differentially expressed genes $(P<0.05$ and fold change $>1.4)$. The $x$ axis (log scale) corresponds to the binomial raw $P$ values. Bottom: ingenuity regulator analysis (see Methods) in KDM2B-overexpressing KRAS-driven leukemias. Regulators with $z$ scores greater than 2 and $P$ overlap values of less than 0.01 are shown.

without necessarily forming physical complexes - characterized by shared chromatin states and related molecular functions (46). We took advantage of the ENCODE project to directly compare the binding pattern of KDM2B with the chromatin occupancy of 125 epigenetic regulators, transcription factors, and chromatin modifications in $\mathrm{K} 562$ cells. Computation of individual binding strengths on the promoters followed by hierarchical clustering revealed a bimodal binding pattern in which approximately $30 \%$ of KDM2B targets were cobound by PRC1/2 and approximately $60 \%$ were cobound by c-MYC/TrxG; no overlap was observed between these modules (Figure 10, A and B, and Supplemental Figure 7, G and H). Coimmunoprecipitation studies confirmed KDM2B interacts with several PcG proteins $(19,26,39,47)$; no interaction was detected between KDM2B and c-MYC/TrxG proteins (Supplemental Figure 7I). Stratification of gene expression and integration with the binding strength of transcription factors showed that, in the context of PRC1/2, KDM2B bound silenced genes enriched for H3K27me3, whereas in the context of c-MYC/TrxG, it bound actively transcribed genes enriched for H3K4me3 and H3K27ac (Supplemental Figure 7J). This analysis also revealed that several factors bind chromatin in a mutually exclusive manner with KDM2B, including several components of RNA Pol III (POLR3A/G, BRF1/2, and BDP1) and SWI/SNF5 (SMARCA4, SMARCB1) chromatinremodeling complex (Supplemental Figure 7J), which antagonizes
KDM2B and PRC2 (Figure 6E, Figure 8C, and ref. 38). Finally, integration of the KDM2B-binding profile with the annotated epigenetic status of chromatin in K562 cells (48) confirmed its association with both repressed and active chromatin states (Figure 10C). IPA showed that genes cobound by KDM2B and PRC1/2 were enriched in WNT signaling and pluripotency networks, while genes cobound by KDM2B and c-MYC/TrxG were enriched in cell-cycle regulation as well as $\mathrm{B}$ and $\mathrm{T}$ cell receptor and cytokine-signaling networks (Figure 10D). Accordingly, analysis of DND41 (T-ALL) cells showed that KDM2B binds active genes - enriched for H3K4me3 - involved in cell cycle, $\mathrm{T}$ cell receptor, and interferon signaling as well as repressed or poised genes - enriched for $\mathrm{H} 3 \mathrm{~K} 27 \mathrm{me} 3$ or bivalent promoters, respectively - involved in canonical and noncanonical (planar cell polarity) Wnt signaling (Supplemental Figure 7K). We conclude that KDM2B modulates the transcriptional outcome of PcG and TrxG complexes to determine cell identity in normal hematopoiesis and leukemias (Figure 10E).

\section{Discussion}

Jumonji domain histone demethylases regulate cellular homeostasis by reversing the degree of methylation on nucleosomes. Mounting evidence indicates that enzymes in this family not only play important roles in cancer, but are also emerging as attractive candidates for therapeutic targeting by small molecule inhibitors. Here, 
A

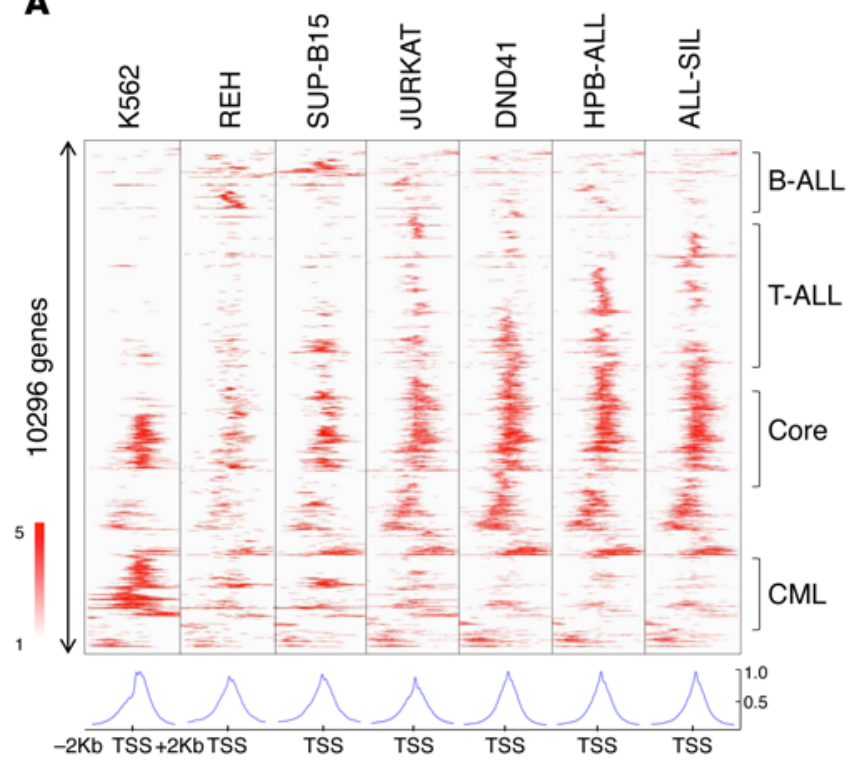

B

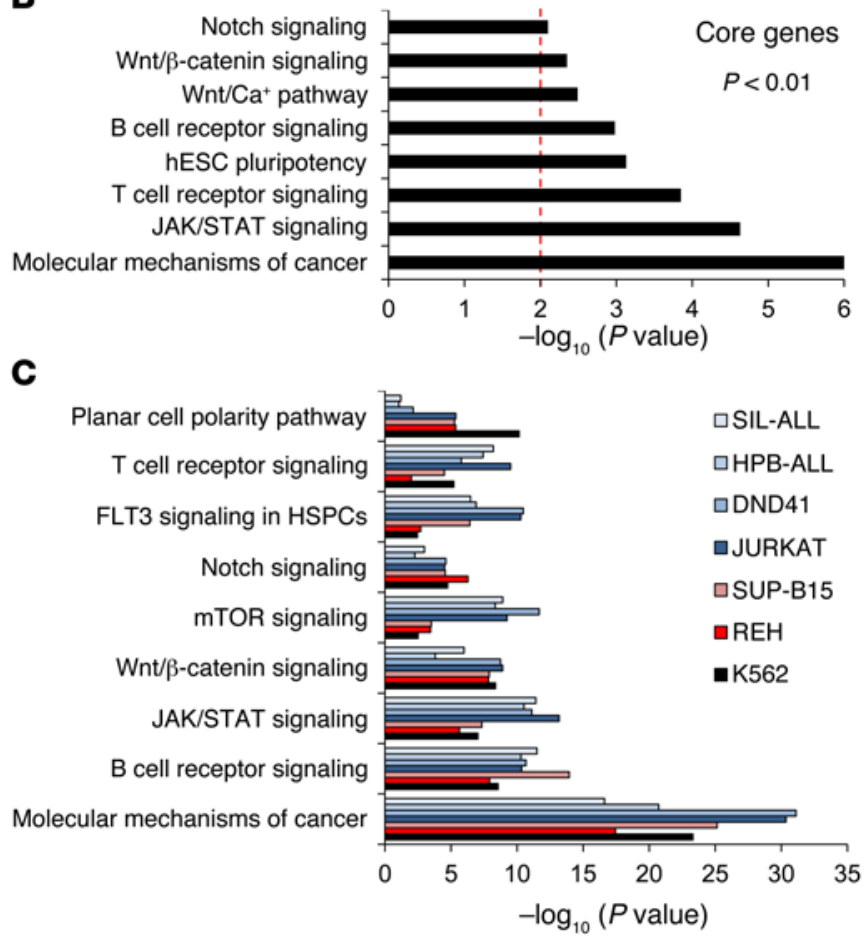

Figure 9. KDM2B regulates cell fate and lineage commitment pathways. (A) Combinatorial binding profiles of KDM2B in 7 human leukemia cell lines. Each horizontal line represents the signal for a gene over the TSS. A $\pm 2 \mathrm{~kb}$ window is shown for each gene. Color bar shows average log 2 ChIP-seq signal intensity. Bottom: metagene analysis of the $\log _{2}$ ChIP-seq signal intensity over the TSS. (B and C) IPA of KDM2B-bound genes (B) in all cell lines (core genes), and (C) in individual cell lines. The $x$ axis (log scale) corresponds to the binomial raw $P$ values.

we describe a conditional KO mouse model of $K d m 2 b$, a histone H3K36 di-demethylase, which regulates cell fate and developmental pathways important in stem cell biology and cancer $(19,22,23$, 25-27, 47). Unlike previous designs that either (a) deleted only the JmjC-containing long isoform in the germline (49), (b) generated a mutant protein by deleting the CXXC domain $\left(K d m 2 b^{4 C X X C}\right)(47)$, or (c) employed a gene trap to delete the long and short isoforms of $K d m 2 b$ without conditional potential (50), our approach deleted all protein coding isoforms of $K d m 2 b$ with conditional potential. We found that deletion of $K d m 2 b$ early in development caused severe growth defects, particularly in the brain, including failure of neural tube closure and craniofacial malformations, which led to lethality before E12.5-E13.5. Contrary to a previous report in which only the long isoform was deleted (49), full penetrance was observed; this suggests the short isoform is equally important in development. The $K d m 2 b^{b x}$ allele was fully recessive, indicating the dominant phenotype described for $K d m 2 b^{W T / A C X X C}$ animals (47) may relate to a neomorphic function of the truncated protein.

We proceeded to investigate the role of $K d m 2 b$ in hematopoiesis based on observations that (a) null embryos were pale and lacked discernible aorta and heart and (b) $K d m 2 b$ is highly expressed in the hemogenic endothelium of E10.5 WT embryos (Figure 1). To this end, we used Tie2-Cre and Vav1-Cre mice to excise the endogenous protein in the hemogenic endothelium and hematopoietic cells, respectively. Tie2-Cre Kdm2 $b^{\text {ffll }}$ embryos showed normal organogenesis at E11.5, but lacked visible aorta and hematopoietic progenitors (VE-cadherin ${ }^{+} \mathrm{CD} 45^{+}$), causing embryonic lethality due to impaired definitive hematopoiesis. Although
Vav1-Cre Kdm $2 b^{A / f}$ mice were viable, they showed a 5-fold reduction in the number of LT-HSCs and defective lymphopoiesis. A similar phenotype was also observed in $\mathrm{M} \times 1$-Cre $\mathrm{Kdm} 2 b^{f / A l}$ mice upon $p I p C$ administration. Transplantation experiments revealed that the effects of Kdm2b in HSPC maintenance and lymphopoiesis are cell intrinsic (Figure 4). Strikingly, a similar phenotype has been reported for Tie2-Cre Ezh $2^{\text {t/fl }}$ embryos, which also lacked definitive hematopoiesis and failed to develop beyond E13.5-E14.5, and in adult mice, Ezh2 was indispensable for lymphopoiesis (5153). Similarly, deletion of Suz12 led to a rapid exhaustion of HSPCs and also impaired lymphopoiesis (54). Interestingly, Kdm2b, Ezh2, and Suz12 are all dispensable for myeloid differentiation. Together, these data suggest that $\mathrm{Kdm} 2 \mathrm{~b}$ and PRC2 regulate key, and largely overlapping, aspects of lymphoid differentiation.

There is no consensus regarding the role of PRC2 in hematopoietic malignancies, as evidence supports both tumor-suppressor and oncogenic functions (55). The human EZH2 gene is located at chromosome 7q, a region that is often deleted in MDS. Loss-offunction mutations in EZH2 and SUZ12 have been identified in AML, suggesting that PRC2 acts as a tumor suppressor in myeloid malignancies $(14,15,56,57)$. Intriguingly, inactivation of PRC2 occurs in almost half of early $\mathrm{T}$ cell precursor acute lymphoblastic leukemias (ETP-ALL), a subset of childhood T-ALL with poor prognosis (11). The common denominator in those malignancies, including ETP-ALL, whose mutational and transcriptional profile is similar to that of myeloid leukemias (11), is activation of the RAS/ERK pathway through direct (gain-of-function mutations, such as in JMML; ref. 14) or indirect (mutations in FLT3 and c-KIT 
A

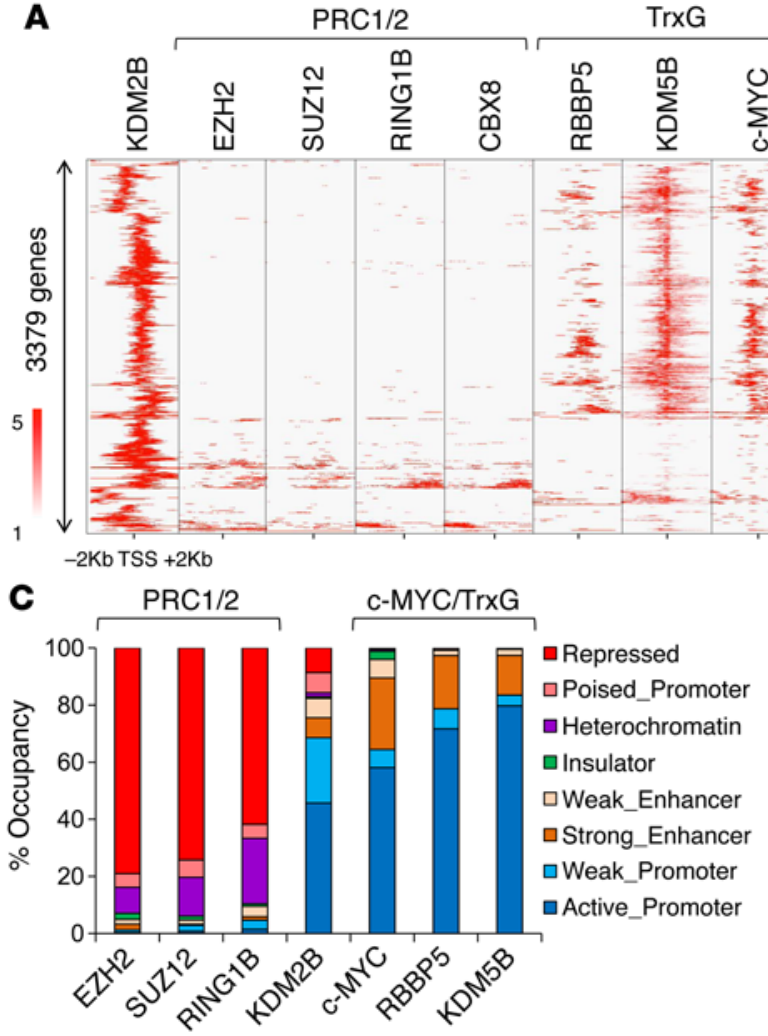

B

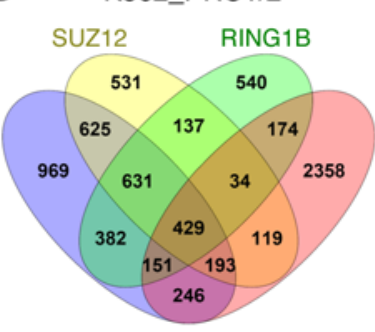

$\mathrm{EZH} 2$

K562 PRC1/2

KDM2B

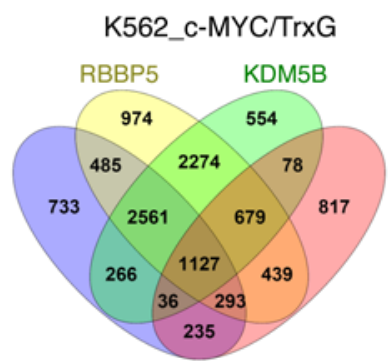

c-MYC

KDM2B

\section{E}

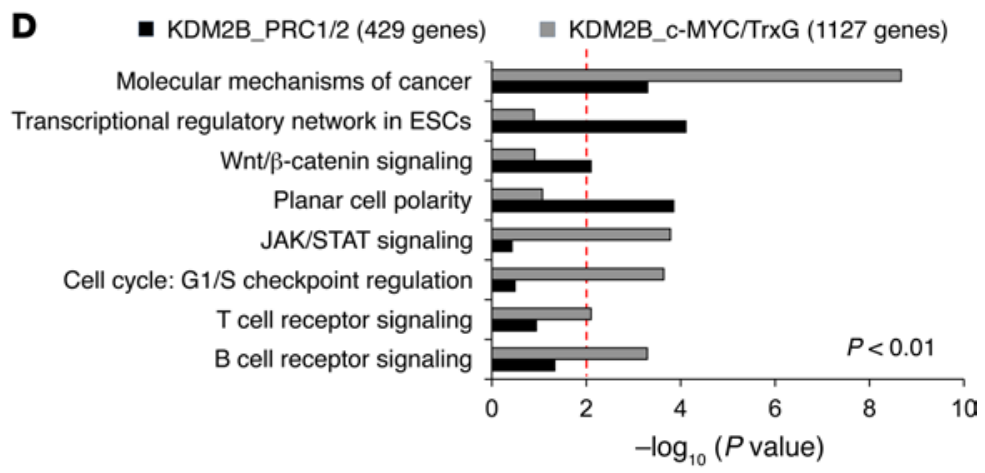

PRC1/2

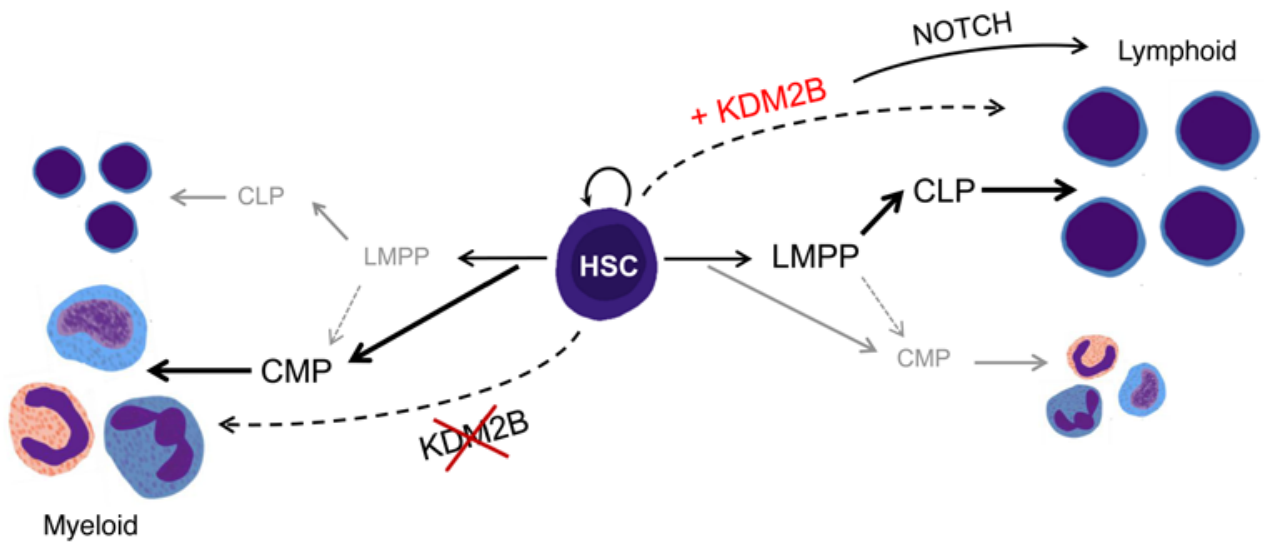

Figure 10. KDM2B crosstalks with PcG and TrxG complexes. (A) Combinatorial binding profiles of KDM2B, PcG, and TrxG proteins in K562 cells. Each hori-

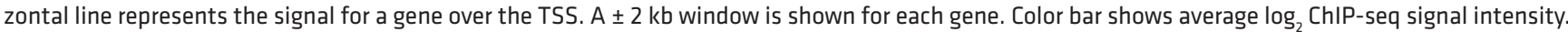
(B) Venn diagrams showing the overlap of KDM2B-bound genes with components of PRC1/2 (left) and c-MYC/TrxC (right) in K562 cells. (C) Stacked bar graph showing the functional annotation of chromatin occupied (\%) by KDM2B, PRC1/2, and TrxG components in K562 cells. (D) IPA of KDM2B-bound genes in the context of PRC1/2 and c-MYC/TrxG. The $x$ axis (log scale) corresponds to the binomial raw $P$ values. (E) Model of KDM2B in hematopoiesis.

receptors) mechanisms. Inactivation of PRC2 also occurs in adult T-ALL in the context of NOTCH1 mutations $(12,13)$. On the other hand, gain-of-function mutations of EZH2 are frequently present in follicular and diffuse large B cell lymphomas, suggesting a prooncogenic role in lymphoid malignancies (16-18).

Here, we extend those findings by showing that KDM2B, which physically interacts with PRC1/2 (Supplemental Figure 7I and refs. 22, 26, 39, 47), can function either as a tumor suppressor or an oncogene, depending on the cellular context. Deletion of $K d m 2 b$ accelerated KRAS-driven myeloid transformation, whereas its overexpression counteracted this oncogenic driver by restoring lymphoid differentiation (Figures 7 and 8 ). Although
KDM2B is not mutated in hematopoietic malignancies, its interacting protein BCOR is frequently inactivated in myeloid malignancies and confers poor prognosis (40). On the contrary, KDM2B is required for MoMuLV-induced T cell lymphomas (19, 20), and, more recently, overexpression of KDM2B in murine BM Sca- $1^{+}$ cells caused long latency mixed lineage leukemias with complete penetrance (58). Although we did not observe leukemias in adult mice following ectopic expression of KDM2B at up to 20 weeks, we did observe expansion of $\operatorname{Lin}^{\text {neg }} \mathrm{KS}^{+}$, CLPs, and LMPPs, an event that could set the stage for malignant transformation in the context of the appropriate oncogenic driver. Consistently, KD of KDM2B ceased proliferation in several human leukemic cell lines 
and abolished their metastatic potential upon injection into NSG mice (Figure 6). A conjecture that could explain this multifaceted role is that KDM2B regulates lineage-specific transcriptional programs through interactions with distinct epigenetic machineries that can be hijacked in oncogenesis. Indeed, ChIP-seq analysis revealed that in lymphoid malignancies (T- and B-ALLs), KDM2B targets were enriched for c-MYC/TrxG-bound genes, whereas in myeloid malignancies (CML), they were enriched for PRC1/2bound genes (Figure 10 and Supplemental Figure 7C). No overlap was observed between those modules, consistent with the finding that mutations in PRC2 components are absent in MLLrearranged leukemias (9). Although it is counterintuitive to associate with both PcG-repressing and c-MYC/TrxG-activating epigenetic networks, the function of KDM2B may be to orchestrate interconnected and interdependent developmental programs in a lineage-specific manner. Since in vivo studies indicate both JmjC domain-dependent and -independent effects, a critical issue will be defining the functions of KDM2B attributed to H3K36 di-demethylase activity. For instance, deletion of both the long and short isoforms (herein) caused severe developmental defects and embryonic lethality compared with the mild phenotype in mice that only lacked the long isoform (49). Along the same lines, we show that the JmjC domain is required for the expansion of $\mathrm{Lin}^{\text {neg }} \mathrm{KS}^{+}$, CLPs, LMPPs, and T lymphocytes (Figure 3). On the other hand, $K d m 2 b^{4 C X X C}$ mutant mice exhibited distinct development defects, suggesting JmjC domain-independent functions, likely due to mistargeting of PRC complexes to chromatin (47). Unlike H3K36me3, which is enriched within gene bodies and plays a role in transcriptional elongation, the distribution and role of H3K36me2 remain elusive. Although we have tested several commercially available antibodies against this histone modification, we failed to validate one suitable for ChIP-seq experiments. Ongoing efforts to generate demethylation-deficient mice through genomic engineering of the endogenous $K d m 2 b$ locus will be useful in addressing this question.

At the molecular level, analysis of gene-expression changes in HSPCs and ChIP-seq studies in human leukemia cell lines revealed a pleiotropic role for KDM2B in differentiation, quiescence, and lymphoid lineage specification through modulation of $\mathrm{NOTCH}$, WNT, and interferon signaling; the latter not only regulates antiviral responses, but also plays important roles in lymphoid commitment and maturation of $\mathrm{T}$ cells $(35,36)$. We discovered that KDM2B sustains NOTCH signaling in HSPCs and T-ALL, a leukemia known to harbor constitutive NOTCH activation (59). Furthermore, NOTCH signaling is required for definitive hematopoiesis and lymphopoiesis, particularly T cell specification, mirroring our findings as KDM2B (a) ectopic expression expanded the T cell compartment and (b) deletion impaired lymphoid development (Figures 2-4). Interestingly, NOTCH functions as a tumor suppressor in myeloid malignancies (60), consistent with our findings that KDM2B restrains KRAS-driven AML. We also found that KDM2B represses WNT signaling through direct transcriptional regulation of several ligands. Intriguingly, recent studies have shown that KDM2B directly demethylates $\beta$-catenin to regulate its stability and dampens WNT singling (61). Downregulation of $\mathrm{Kdm} 2 \mathrm{~b}$ in Xenopus (61), zebrafish (Supplemental Figure 1, F and G, ref. 30), and mice (this study) caused embryonic lethality due to severe developmental defects and compromised the development of the hematopoietic system, indicating an evolutionary conserved role. Given that KDM2B is a potential target for cancer therapy, the consequences of its inhibition need to be carefully considered, as they may be contingent on lineage-specific effects. Although further studies are needed to delineate the complexity of those circuitries, it is becoming evident that restoring differentiation in leukemias is a promising therapeutic approach.

\section{Methods}

BM transplantations. B6.SJL-Ptprca Pep3b/BoyJ (CD45.1; stock number 002014) mice were purchased from the Jackson Laboratory. $K d m 2 b^{f / f l}$ mice were backcrossed with C57BL/6 (CD45.2) for 6 generations. For noncompetitive transplantation, $\mathrm{BM}$ cells isolated from Vav1-Cre Kdm2 $b^{+/+}$or Vav1-Cre Kdm2 $b^{f / f l} \mathrm{CD} 45.2$ animals were injected through the tail vein into CD45.1 recipients that received 1 dose of 9.5 Gy of total body irradiation. For competitive transplantation, total BM isolated from $\mathrm{M} x 1$-Cre $\mathrm{Kdm} 2 \mathrm{~b}^{+/+}$or $\mathrm{M} x 1$-Cre $\mathrm{Kdm} 2 \mathrm{~b}^{f / f l} \mathrm{CD} 45.2$ animals was mixed in a 1:1 ratio with CD 45.1 BM cells and injected through the tail vein into CD45.1 recipients that received 1 dose of $9.5 \mathrm{~Gy}$ of total body irradiation. After confirming successful reconstitution by means of flow cytometry, excision was achieved by intraperitoneal injection of pIpC (GE Healthcare) at a dose of $8.5 \mathrm{mg} / \mathrm{kg}$ every other day for 4 days.

Flow cytometry. All immunostains were performed as we have described previously $(5,62,63)$. To stain peripheral blood cells, erythrocytes were first lysed in ammonium chloride-potassium bicarbonate (AKC) buffer, and cells were washed with PBS and resuspended in staining buffer ( $5 \%$ heat-inactivated calf serum, $1 \%$ penicillin/streptomycin in PBS). Cells were stained with fluorochrome-conjugated antibodies against B cell marker B220 (clone RA3-682), T cell marker CD3e (clone 145-2c11), neutrophil marker Gr-1 (clone RB6-85C), and monocyte marker CD11b (clone M1/70). In transplantation experiments, blood chimerism was determined using fluorochrome-conjugated antibodies against CD45.1 (clone A20; host cells) and CD45.2 (clone 104; donor cells). For BM staining, femurs and tibias were crushed with a sterile mortar and pestle into media and filtered through a 40- $\mu \mathrm{m}$ mesh. BM was depleted of terminally differentiated cells (lineage positive) by using a cocktail of conjugated antibodies against all hematopoietic lineages (Gr-1, CD11b, B220, CD3e, Ter-119) using either (a) biotin/streptavidin-coupled magnetic bead separation (Life Technologies) or (b) fluorochrome-conjugated antibodies and separation by gating. Briefly, $5 \times 10^{6}$ cells were stained with c-Kit (clone 2B8) and Sca-1 (clone D7) antibodies; CD48 (clone HM48-1) and CD150 (clone TC15-12F12.2) were included for LT-HSC, FLT3 (clone A2F10) for LMPP, IL-7R $\alpha$ (clone A7R34) for CLP, and CD16/CD32 (clone 93) and CD34 (clone RAM34) for CMP, GMP, and MEP staining. For BM intracellular staining, IKAROS (clone 2A9/Ikaros) and PAX5 (clone 1H9) were used in combination with the Nuclear Factor Fixation and Permeabilization Kit (BioLegend). For cell-cycle analysis, first BM cells were stained for lineage/c-Kit/Sca-1, then fixed and permeabilized with the Nuclear Factor Fixation and Permeabilization Kit and sequentially stained with $1 \mu \mathrm{g} / \mathrm{ml}$ DAPI (LifeTechnologies) and $5 \mu \mathrm{M}$ Pyronin-Y (Sigma-Aldrich). To inhibit efflux, Verapamil (Sigma-Aldrich) was added at a final concentration of $50 \mu \mathrm{M}$. For AGM staining, single-cell suspensions were prepared and stained with fluorochrome-conjugated antibodies against VE-Cadherin (BV13) and CD45 (30-F11). All antibodies were purchased from BioLegend and eBioScience, and clone 
numbers are indicated in parentheses. Data collection took place on a BD FACSCalibur DxP8 Analyzer or BD FACSAria Sorter, and analyses were performed with FlowJo software (TreeStar).

Gene-expression analysis. For RNA-seq, approximately $5 \times 10^{4} \mathrm{Lin}^{\text {neg }}$ $\mathrm{KS}^{+}$cells were sorted from Vav1-Cre Kdm2 $b^{+/+}$and Vav1-Cre Kdm $2 b^{\text {fl/fl }}$ mice and cultured in IMDM supplemented with $10 \%$ fetal bovine serum, $10 \mathrm{ng} / \mathrm{ml} \mathrm{FLT3}$ ligand, $50 \mathrm{ng} / \mathrm{ml} \mathrm{SCF}, 10 \mathrm{ng} / \mathrm{ml} \mathrm{IL}-6,10 \mathrm{ng} / \mathrm{ml} \mathrm{IL}-3$, and $20 \mathrm{ng} / \mathrm{ml}$ TPO for 36 hours. RNA was extracted using a RNeasy Mini Kit (QIAGEN). Two biological replicates were generated from WT and $K d m 2 b$-null Lin ${ }^{\text {neg }} \mathrm{KS}^{+}$cells and sequenced with an Illumina Hi-Seq 2500 instrument $(2 \times 100$, paired end). FastQC was used to assess the quality of the reads. Tophat (v2.0.12, ref. 64) was used to align the FastQC files to $\mathrm{mm} 9$ genome assembly, with default parameters. Differentially expressed genes were identified by Cuffdiff from Cufflinks software (v2.2.1, ref. 65). As shown in Supplemental Figure 5C, RNA-seq data for 81 leukemia cell lines were downloaded from the Cancer Genomics Hub (cghub, https://browser.cghub.ucsc.edu/search/). Cuffquant from Cufflinks (v2.2.1) was used to quantitate the gene-expression values, and Cuffnorm was used to normalize across the 81 cell lines. The similarity matrix (Spearman's rank correlation coefficient) and heat map were generated with Partek Genomics Suite software, version 6.6. Affymetrix gene-expression profiling and analysis were as follows: for human leukemia cell lines (JURKAT, ALL-SIL, DND41, and K562), total mRNA was isolated with the RNeasy Mini Kit (QIAGEN) from cells 6 days after infection with shKDM2B and subsequent puromycin selection. For murine leukemias, BM was harvested and depleted of lineage committed cells as described in the flow cytometry section. Preparation of cRNA and hybridization and scanning of microarrays were performed according to the manufacturer's protocol (Affymetrix) in house. In brief, $100 \mathrm{ng}$ of total RNA was used as input for the PrimeView (3'IVT human array) and the MoGene 2.0 ST (mouse whole transcript array) microarrays (Affymetrix). Hybridization was performed using a GeneChip Hybridization Oven 640 overnight at $45^{\circ} \mathrm{C}$. Microarray washing and staining were done using a GeneChip Fluidics Station 450 and were scanned using the GeneChip Scanner 3000 7G (Affymetrix), commanded by Affymetrix GeneChip Command Console Software. Probe level analysis including background subtraction and quantile normalization took place with the Robust Multi Array Average Algorithm (RMA) using Affymetrix Expression Console Software 1.3. Differentially expressed genes $(P<$ 0.05 and fold change $>1.5$ for the PrimeView and $>1.4$ for the MoGene 2.0 ST exon array) were determined using the Transcriptome Analysis Console v3.0. Raw and processed RNA-seq and Affymetrix data were deposited in the NCBI's Gene Expression Omnibus (GEO GSE70729).
Methylcellulose colony-forming assays. BM cells were plated in $2 \mathrm{ml}$ of MethoCult M3434 or M3630 (Stem Cell Technologies) in duplicate on a 6-well plate and cultured at $37^{\circ} \mathrm{C}$ for 10 days before scoring for colony formation.

Statistics. Data are presented as mean \pm SEM. Significance was analyzed using 2-tailed Student's $t$ test. A $P$ value of less than 0.05 was considered statistically significant. To compare more than 2 experimental groups, 1-way ANOVA was used. In the Kaplan-Meier plots, the log-rank (Mantel-Cox) test was used to compare survival between experimental groups. Statistical analysis took place using GraphPad PRISM version 5.01. Survival analysis of the LAML TCGA cohort was performed with the Prediction of Clinical Outcomes from Genomic profiles (PRECOG software, https://precog.stanford.edu/; ref. 42).

Study approval. All animal experiments were conducted under protocols A292 and A293, which were reviewed and approved by the Institutional Animal Care and Use Committee of GWU.

Additional experimental procedures are presented in Supplemental Methods.

\section{Author contributions}

JA and AT conceived the project and performed the experiments. AF helped with flow cytometry analysis and provided advice for mouse experiments. YK, WP, and AT performed bioinformatics analysis. All authors contributed to experimental design and wrote the paper. AT supervised the project. All authors discussed the results and commented on the manuscript.

\section{Acknowledgments}

We thank R. Hawley, I. Riz, and T. Hawley (GWU) for advice and technical support, N. Bardeesy (Massachusetts General Hospital) for advice in generating transgenic mice, and A. Popratiloff (GWU Center for Microscopy and Image Analysis, supported by NCRR SIG 1S10RR025565). This research was supported by the Vaincre le Cancer-nrb grant and the ATIP-Avenir Young Investigator Program (to A. Foudi), a National Institute of Allergy and Infection Diseases grant (R21AI113806 to W. Peng), an Alex's Lemonade Stand Foundation Young Investigator Award, and National Cancer Institute grants (R00CA158582 and R21CA182662 to A. Tzatsos).

Address correspondence to: Alexandros Tzatsos, Cancer Epigenetics Laboratory, George Washington University, 2300 I Street NW, Ross Hall 226E, Washington, DC 20037, USA. Phone: 202.994.9104; E-mail: atzatsos@gwu.edu.
1. Kiel MJ, Yilmaz OH, Iwashita T, Terhorst C, Morrison SJ. SLAM family receptors distinguish hematopoietic stem and progenitor cells and reveal endothelial niches for stem cells. Cell. 2005;121(7):1109-1121.

2. McKinney-Freeman $S$, et al. The transcriptional landscape of hematopoietic stem cell ontogeny. Cell Stem Cell. 2012;11(5):701-714.

3. Orkin SH, Zon LI. Hematopoiesis: an evolving paradigm for stem cell biology. Cell. 2008;132(4):631-644.

4. Dzierzak E, Speck NA. Of lineage and legacy: the development of mammalian hematopoietic stem cells. Nat Immunol. 2008;9(2):129-136.
5. Foudi A, et al. Analysis of histone 2B-GFP retention reveals slowly cycling hematopoietic stem cells. Nat Biotechnol. 2009;27(1):84-90.

6. Wilson A, et al. Hematopoietic stem cells reversibly switch from dormancy to selfrenewal during homeostasis and repair. Cell. 2008;135(6):1118-1129.

7. Schuettengruber B, Chourrout D, Vervoort M, Leblanc B, Cavalli G. Genome regulation by polycomb and trithorax proteins. Cell. 2007;128(4):735-745.

8. Gao Z, et al. PCGF homologs, CBX proteins, and RYBP define functionally distinct PRC1 family complexes. Mol Cell. 2012;45(3):344-356.
9. Meyer C, et al. The MLL recombinome of acute leukemias in 2013. Leukemia. 2013;27(11):2165-2176.

10. Lindsley RC, et al. Acute myeloid leukemia ontogeny is defined by distinct somatic mutations. Blood. 2015;125(9):1367-1376.

11. Zhang J, et al. The genetic basis of early T-cell precursor acute lymphoblastic leukaemia. Nature. 2012;481(7380):157-163.

12. Ntziachristos $P$, et al. Genetic inactivation of the polycomb repressive complex 2 in $\mathrm{T}$ cell acute lymphoblastic leukemia. Nat Med. 2012;18(2):298-301.

13. Simon C, et al. A key role for EZH2 and associ- 
ated genes in mouse and human adult T-cell acute leukemia. Genes Dev. 2012;26(7):651-656.

14. Caye A, et al. Juvenile myelomonocytic leukemia displays mutations in components of the RAS pathway and the PRC2 network. Nat Genet. 2015;47(11):1334-1340.

15. Cancer Genome Atlas Research Network. Genomic and epigenomic landscapes of adult de novo acute myeloid leukemia. $N$ Engl J Med. 2013;368(22):2059-2074.

16. Sneeringer CJ, et al. Coordinated activities of wild-type plus mutant EZH2 drive tumor-associated hypertrimethylation of lysine 27 on histone H3 (H3K27) in human B-cell lymphomas. Proc Natl Acad Sci U S A. 2010;107(49):20980-20985.

17. Morin RD, et al. Somatic mutations altering $\mathrm{EZH} 2$ (Tyr641) in follicular diffuse large B-cell lymphomas of germinal-center origin. Nat Genet. 2010;42(2):181-185.

18. Yap DB, et al. Somatic mutations at EZH2 Y641 act dominantly through a mechanism of selectively altered PRC2 catalytic activity, to increase H3K27 trimethylation. Blood. 2011;117(8):2451-2459.

19. Tzatsos A, Pfau R, Kampranis SC, Tsichlis PN. Ndy1/KDM2B immortalizes mouse embryonic fibroblasts by repressing the Ink4a/Arf locus. Proc Natl Acad Sci U S A. 2009;106(8):2641-2646.

20. Pfau R, Tzatsos A, Kampranis SC, Serebrennikova OB, Bear SE, Tsichlis PN. Members of a family of JmjC domain-containing oncoproteins immortalize embryonic fibroblasts via a JmjC domain-dependent process. Proc Natl Acad Sci U S A. 2008;105(6):1907-1912.

21. He J, Nguyen AT, Zhang Y. KDM2b/JHDM1b, an $\mathrm{H} 3 \mathrm{~K} 36 \mathrm{me} 2$-specific demethylase, is required for initiation maintenance of acute myeloid leukemia. Blood. 2011;117(14):3869-3880.

22. Tzatsos A, et al. KDM2B promotes pancreatic cancer via Polycomb-dependent -independent transcriptional programs. JClin Invest. 2013;123(2):727-739.

23. Tzatsos A, et al. Lysine-specific demethylase 2B (KDM2B)-let-7-enhancer of zester homolog 2 (EZH2) pathway regulates cell cycle progression senescence in primary cells. J Biol Chem. 2011;286(38):33061-33069.

24. Wang $\mathrm{T}$, et al. The histone demethylases Jhdm1a/1b enhance somatic cell reprogramming in a vitamin-C-dependent manner. Cell Stem Cell. 2011;9(6):575-587.

25. Liang G, He J, Zhang Y. Kdm2b promotes induced pluripotent stem cell generation by facilitating gene activation early in reprogramming. Nat Cell Biol. 2012;14(5):457-466.

26. Wu X, Johansen JV, Helin K. Fbxl10/Kdm2b recruits polycomb repressive complex 1 to $\mathrm{CpG}$ islands regulates $\mathrm{H} 2 \mathrm{~A}$ ubiquitylation. Mol Cell. 2013;49(6):1134-1146.

27. He J, Shen L, Wan M, Taranova O, Wu H, Zhang Y. Kdm2b maintains murine embryonic stem cell status by recruiting PRC1 complex to CpG islands of developmental genes. Nat Cell Biol. 2013;15(4):373-384.

28. Mao X, Fujiwara Y, Orkin SH. Improved reporter strain for monitoring Cre recombinase-mediated DNA excisions in mice. Proc Natl Acad Sci U S A. 1999;96(9):5037-5042.
29. Koni PA, Joshi SK, Temann UA, Olson D, Burkly L, Flavell RA. Conditional vascular cell adhesion molecule 1 deletion in mice: impaired lymphocyte migration to bone marrow. J Exp Med. 2001;193(6):741-754.

30. Huang HT, et al. A network of epigenetic regulators guides developmental haematopoiesis in vivo. Nat Cell Biol. 2013;15(12):1516-1525.

31. Stadtfeld M, Graf T. Assessing the role of hematopoietic plasticity for endothelial and hepatocyte development by non-invasive lineage tracing. Development. 2005;132(1):203-213.

32. Adolfsson J, et al. Identification of Flt $3^{+}$lymphomyeloid stem cells lacking erythro-megakaryocytic potential a revised road map for adult blood lineage commitment. Cell. 2005;121(2):295-306.

33. Bracken AP, Dietrich N, Pasini D, Hansen KH, Helin K. Genome-wide mapping of Polycomb target genes unravels their roles in cell fate transitions. Genes Dev. 2006;20(9):1123-1136.

34. Zhou BO, Ding L, Morrison SJ. Hematopoietic stem and progenitor cells regulate the regeneration of their niche by secreting Angiopoietin-1. Elife. 2015; 4:e05521.

35. Xu H, et al. Regulation of bifurcating B cell trajectories by mutual antagonism between transcription factors IRF4 and IRF8. Nat Immunol. 2015;16(12):1274-1281.

36. Wang S, He Q, Ma D, Xue Y, Liu F. Irf4 regulates the choice between $\mathrm{T}$ lymphoid-primed progenitor myeloid lineage fates during embryogenesis. Dev Cell. 2015;34(6):621-631.

37. Barretina J, et al. The Cancer Cell Line Encyclopedia enables predictive modelling of anticancer drug sensitivity. Nature. 2012;483(7391):603-607.

38. Wilson BG, et al. Epigenetic antagonism between polycomb and SWI/SNF complexes during oncogenic transformation. Cancer Cell. 2010;18(4):316-328.

39. Gearhart MD, Corcoran CM, Wamstad JA, Bardwell VJ. Polycomb group and SCF ubiquitin ligases are found in a novel BCOR complex that is recruited to BCL6 targets. Mol Cell Biol. 2006;26(18):6880-6889.

40. Grossmann V, et al. Whole-exome sequencing identifies somatic mutations of BCOR in acute myeloid leukemia with normal karyotype. Blood. 2011;118(23):6153-6163.

41. Huether R, et al. The landscape of somatic mutations in epigenetic regulators across 1,000 paediatric cancer genomes. Nat Commun. 2014;5:3630

42. Gentles AJ, et al. The prognostic landscape of genes and infiltrating immune cells across human cancers. Nat Med. 2015;21(8):938-945.

43. Zhao Z, et al. p53 loss promotes acute myeloid leukemia by enabling aberrant self-renewal. Genes Dev. 2010;24(13):1389-1402.

44. McLean CY, et al. GREAT improves functional interpretation of cis-regulatory regions. Nat Biotechnol. 2010;28(5):495-501.

45. Herranz D, et al. A NOTCH1-driven MYC enhancer promotes $\mathrm{T}$ cell development, transformation acute lymphoblastic leukemia. Nat Med. 2014;20(10):1130-1137.

46. Ram O, et al. Combinatorial patterning of chromatin regulators uncovered by genome- wide location analysis in human cells. Cell. 2011;147(7):1628-1639.

47. Blackledge NP, et al. Variant PRC1 complexdependent H2A ubiquitylation drives PRC2 recruitment polycomb domain formation. Cell. 2014;157(6):1445-1459.

48. Ernst J, Kellis M. ChromHMM: automating chromatin-state discovery and characterization. Nat Methods. 2012;9(3):215-216.

49. Fukuda T, Tokunaga A, Sakamoto R, Yoshida N. Fbxl10/Kdm2b deficiency accelerates neural progenitor cell death leads to exencephaly. $\mathrm{Mol}$ Cell Neurosci. 2011;46(3):614-624.

50. Boulard M, Edwards JR, Bestor TH. FBXL10 protects Polycomb-bound genes from hypermethylation. Nat Genet. 2015;47(5):479-485.

51. Mochizuki-Kashio M, et al. Dependency on the polycomb gene Ezh2 distinguishes fetal from adult hematopoietic stem cells. Blood. 2011;118(25):6553-6561.

52. Delgado-Olguin P, et al. Ezh2-mediated repression of a transcriptional pathway upstream of Mmp9 maintains integrity of the developing vasculature. Development. 2014;141(23):4610-4617.

53. Su IH, et al. Ezh2 controls B cell development through histone $\mathrm{H} 3$ methylation Igh rearrangement. Nat Immunol. 2003;4(2):124-131.

54. Lee SC, et al. Polycomb repressive complex 2 component Suz12 is required for hematopoietic stem cell function lymphopoiesis. Blood. 2015;126(2):167-175.

55. Hock H. A complex Polycomb issue: the two faces of EZH2 in cancer. Genes Dev. 2012;26(8):751-755.

56. Ernst $\mathrm{T}$, et al. Inactivating mutations of the histone methyltransferase gene EZH2 in myeloid disorders. Nat Genet. 2010;42(8):722-726.

57. Nikoloski G, et al. Somatic mutations of the histone methyltransferase gene EZH2 in myelodysplastic syndromes. Nat Genet. 2010;42(8):665-667.

58. Ueda T, et al. Fbxl10 overexpression in murine hematopoietic stem cells induces leukemia involving metabolic activation and upregulation of Nsg2. Blood. 2015;125(22):3437-3446.

59. Van Vlierberghe P, Ferrando A. The molecular basis of T cell acute lymphoblastic leukemia. J Clin Invest. 2012;122(10):3398-3406.

60. Lobry C, et al. Notch pathway activation targets AML-initiating cell homeostasis and differentiation. J Exp Med. 2013;210(2):301-319.

61. Lu L, et al. Kdm2a/b lysine demethylases regulate canonical Wnt signaling by modulating the stability of nuclear $\beta$-catenin. Dev Cell. 2015;33(6):660-674.

62. Foudi A, et al. Distinct, strict requirements for Gfi-1b in adult bone marrow red cell platelet generation. J Exp Med. 2014;211(5):909-927.

63. Gurumurthy S, et al. The Lkb1 metabolic sensor maintains haematopoietic stem cell survival. Nature. 2010;468(7324):659-663.

64. Trapnell C, Pachter L, Salzberg SL. TopHat: discovering splice junctions with RNA-Seq. Bioinformatics. 2009;25(9):1105-1111.

65. Trapnell C, et al. Transcript assembly and quantification by RNA-Seq reveals unannotated transcripts and isoform switching during cell differentiation. Nat Biotechnol. 2010;28(5):511-515 